Collection SFN 7 (2007) 147-172

(C) EDP Sciences, Les Ulis

DOI: $10.1051 / \mathrm{sfn}: 2007021$

\title{
Polarimétrie neutronique longitudinale et sphérique en diffusion inélastique
}

\author{
L.P. Regnault ${ }^{1}$
}

${ }^{1}$ CEA-Grenoble, Département de Recherche Fondamentale sur la Matière condensée, SPSMS-MDN, 17 rue des Martyrs, 38054 Grenoble Cedex 9, France

Résumé. Dans ce cours, nous allons présenter les principes généraux de l'analyse de polarisation neutronique longitudinale et sphérique, en mettant un accent plus particulier sur le cas de la diffusion inélastique des neutrons. La force de la polarimétrie neutronique en diffusion inélastique sera illustrée sur des exemples caractéristiques choisis dans les domaines du magnétisme de terres-rares anormales, du magnétisme de systèmes de spins classiques et quantiques à basse dimension et de la supraconductivité Ĺ haute température critique.

\section{NOTATIONS}

$\begin{array}{ll}\mathbf{k}_{0} & \left.\text { Vecteur d'onde des neutrons incidents (également noté } \mathbf{k}_{i}\right) \\ \mathbf{k}_{1} & \left.\text { Vecteur d'onde des neutrons diffusés (également noté } \mathbf{k}_{f}\right) \\ \mathbf{Q} & \text { Vecteur de diffusion }\left(\mathbf{Q}=\mathbf{k}_{0}-\mathbf{k}_{1}\right) \\ Q & \text { Module de } \mathbf{Q} \\ E_{0} & \left.\text { Energie des neutrons incidents (également notée } E_{i}\right) \\ E_{1} & \left.\text { Energie des neutrons diffusés (également notée } E_{f}\right) \\ \hbar \omega & \left.\text { Transfert d'énergie ( } \omega=E_{0}-E_{1}\right) \\ \mathbf{H}_{N} & \text { Vecteur du réseau réciproque }\left(\mathbf{H}_{N}=\left(h_{N}, k_{N}, l_{N}\right)\right) \\ \mathbf{P}_{0} & \text { Vecteur polarisation incident } \\ P_{0} & \text { Valeur absolue de la polarisation incidente } \\ P_{0 \alpha} & \alpha^{\text {ieme }} \text { composante de la polarisation incidente } \\ \mathbf{P} & \text { Vecteur polarisation diffusé } \\ P_{\alpha} & \alpha^{\text {ieme }} \text { composante de la polarisation diffusée } \\ P_{\alpha \beta} & \text { Composante } \alpha \beta \text { de la matrice de polarisation } \\ N_{Q} & \text { Opérateur d'intensité nucléaire diffusée pour le vecteur de diffusion } \mathbf{Q} \\ \mathbf{M}_{Q} & \text { Opérateur d'intensité magnétique diffusée pour le vecteur de diffusion } \mathbf{Q} \\ M_{Q \alpha} & \alpha^{\text {ieme }} \text { composante de l'opérateur d'intensité magnétique diffusée } \\ \mathbf{M}_{\perp Q} & \text { Opérateur d'interaction magnétique pour le vecteur de diffusion } \mathbf{Q} \\ M_{\perp Q \alpha} & \alpha^{\text {ieme }} \text { composante de l'opérateur d'interaction magnétique } \\ \mathbf{k} & \text { Vecteur de propagation magnétique }\end{array}$


$S_{\alpha \beta}(\mathbf{q}, \omega) \quad$ Facteur de structure associé aux composantes magnétiques $\alpha$ et $\beta$

$A^{+} \quad$ Adjoint de l'opérateur A

$A^{*} \quad$ Complexe conjugué de A

$\mathbf{R}_{n i} \quad$ Vecteur de position atomique dans la $n^{\text {ieme }}$ cellule du $i^{\text {ieme }}$ sous-réseau de Bravais

$T \quad$ Température de l'échantillon

H Vecteur champ magnétique

$H_{\alpha} \quad \alpha^{\text {ieme }}$ composante de $\mathbf{H}$

\section{INTRODUCTION}

Dans ce chapitre, nous allons aborder les aspects principaux liés à l'utilisation des neutrons polarisés et de l'analyse de polarisation en diffusion inélastique des neutrons. La diffusion inélastique des neutrons non-polarisés est bien connue pour être une technique incontournable pour l'étude des dynamiques de réseau et de spin dans une grande variété de matériaux. Néanmoins, dans certains cas particuliers, les degrès de liberté structuraux et magnétiques donnent lieu à des contributions qui sont soit superposées ou, dans des cas plus compliqués, qui interférent fortement, donnant ainsi naissance à des modes hybrides ou mixtes. Dans ces cas très intéressants, la diffusion inélastique des neutrons standard (i.e. en neutrons non-polarisés) montre ses limites et une méthode plus sophistiquée doit être utilisée pour de résoudre le problème. Comme en diffraction (voir les chapitres précédents), dans de nombreuses études l'utilisation de la diffusion inélastique des neutrons polarisés en association avec une méthode précise d'analyse de polarisation est la seule manière d'obtenir certaines informations pertinantes de façon non-ambigüe. La diffusion inélastique des neutrons polarisés est ainsi essentielle pour tout ce qui concerne les mesures nécessitant la séparation propre des contributions magnétiques et nucléaires, la détermination indépendante des différentes composantes des excitations ou fluctuations magnétiques dynamiques, l'étude des fonctions de corrélations hybrides couplant des degrès de liberté différents (spin-réseau, orbite-réseau), ou identiques (spin-orbite, chiralité).

\section{POLARIMÉTRIE NEUTRONIQUE INÉLATIQUE: THÉORIE ET PRATIQUE}

Le cadre théorique de l'interaction neutron-matière a été développé il y a plusieurs années [1-7]. Dans cette partie, nous allons juste rappeler les principales formules qui nous seront utiles pour la compréhension de la polarimétrie neutronique sur des signaux inélastiques. Les équations générales donnant la polarisation du faisceau de neutrons diffusé et la section efficace différentielle inélastique en fonction de la polarisation incidente, ont été établies au début des années 60 par Maleyev et al. [3,6] et Blume [7]. En appelant $\mathbf{k}_{0}$ et $\mathbf{k}_{1}$ les vecteurs d'onde incident et final, et $\mathbf{P}_{0}$ le vecteur polarisation du faisceau de neutrons incident, la polarisation du faisceau de neutrons après diffusion sur l'échantillon, $\mathbf{P}$, est donnée par l'équation suivante [9-11]:

$$
\begin{aligned}
\mathbf{P}\left(d^{2} \sigma / d \Omega d \omega\right)= & C_{p} \frac{k_{1}}{k_{0}}\left\{\left\langle N_{Q} N_{Q}^{+}\right\rangle_{\omega} \mathbf{P}_{0}+\left\langle\left(\mathbf{P}_{0} \cdot \mathbf{M}_{\perp Q}^{+}\right) \mathbf{M}_{\perp Q}\right\rangle_{\omega}+\left\langle\mathbf{M}_{\perp Q}^{+}\left(\mathbf{P}_{0} \cdot \mathbf{M}_{\perp Q}\right)\right\rangle_{\omega}\right. \\
& -\left\langle\mathbf{M}_{\perp Q} \cdot \mathbf{M}_{\perp Q}^{+}\right\rangle_{\omega} \mathbf{P}_{0}+i\left\langle\mathbf{M}_{\perp Q} \times \mathbf{M}_{\perp Q}^{+}\right\rangle_{\omega} \\
& +\left\langle N_{Q}^{+} \mathbf{M}_{\perp Q}\right\rangle_{\omega}+\left\langle\mathbf{M}_{\perp Q}^{+} N_{Q}\right\rangle_{\omega} \\
& \left.+i \mathbf{P}_{0} \times\left[\left\langle\mathbf{M}_{\perp Q}^{+} N_{Q}\right\rangle_{\omega}-\left\langle N_{Q}^{+} \mathbf{M}_{\perp Q}\right\rangle_{\omega}\right]\right\}
\end{aligned}
$$


où la section efficace inélastique double-différentielle $d^{2} \sigma / d \Omega d \omega$ ) est donnée par :

$$
\begin{aligned}
d^{2} \sigma / d \Omega d \omega=C_{\sigma} & \frac{k_{1}}{k_{0}}\left\{\left\langle N_{Q} N_{Q}^{+}\right\rangle_{\omega}\right. \\
& +\left\langle\mathbf{M}_{\perp Q} \cdot \mathbf{M}_{\perp Q}^{+}\right\rangle_{\omega}-i \mathbf{P}_{0} \cdot\left\langle\mathbf{M}_{\perp Q} \times \mathbf{M}_{\perp Q}^{+}\right\rangle_{\omega} \\
& \left.+\mathbf{P}_{0} \cdot\left[\left\langle N_{Q}^{+} \mathbf{M}_{\perp Q}\right\rangle_{\omega}+\left\langle\mathbf{M}_{\perp Q}^{+} N_{Q}\right\rangle_{\omega}\right]\right\}
\end{aligned}
$$

Dans les équations (3.1) et (3.2), $C_{p}$ et $C_{\sigma}$ sont deux facteurs d'échelle, $\mathbf{Q}=\mathbf{k}_{0}-\mathbf{k}_{1}$ est le vecteur de diffusion (alias transfert de moment des neutrons),

$N_{Q}=N^{1 / 2} \sum_{j} b_{j} e^{i \mathbf{Q} \cdot \mathbf{R}_{j}}$ est l'opérateur (scalaire complexe) d'amplitude de diffusion nucléaire, où $\mathbf{R}_{j}$ et $b_{j}$ sont respectivement le vecteur position et la longueur de diffusion du $j^{\text {ieme }}$ noyau, et $\mathrm{N}$ est le nombre total de noyaux dans le système. Les crochets angulaires dénotent à la fois la moyenne de Gibbs et la moyenne sur les états de spin du faisceau incident. L'opérateur d'interaction magnétique (vecteur à composantes complexes) est défini par la relation habituelle :

$$
\mathbf{M}_{\perp Q}=r_{0} N^{1 / 2} \sum_{j}\left[\mathbf{M}_{j}-\left(\mathbf{Q} \cdot \mathbf{M}_{j}\right) \cdot \mathbf{Q} / Q^{2}\right] e^{i \mathbf{Q} \cdot \mathbf{R}_{j}},
$$

où $r_{0} \approx 0.54 \times 10^{-12} \mathrm{~cm}$ et $\mathbf{M}_{j}=\left[\mathbf{s}_{j}-\frac{i}{\hbar}\left(\mathbf{Q} \times \mathbf{p}_{j}\right) / Q^{2}\right]$ dépend à la fois du spin $\left(\mathbf{s}_{j}\right)$ et de l'impulsion $\left(\mathbf{p}_{j}\right)$ électronique [12-14]. En particulier, le dernier terme permet en principe de sonder (au moins partiellement) les degrés de liberté orbitaux. Comme il est bien connu, seules les composantes de $\mathbf{M}_{\mathbf{Q}}$ perpendiculaires au vecteur de diffusion $\mathbf{Q}$ contribuent au processus de diffusion magnétique. Les équations (3.1) et (3.2) contiennent plusieurs termes de nature différente : $\left\langle N_{Q} N_{Q}^{+}\right\rangle_{\omega}$ correspond aux fonctions de corrélation purement nucléaires et ne modifie pas la polarisation initiale, qui est conservée dans le processus de diffusion. Les deux termes suivant $\left\langle\left(\mathbf{P}_{0} \cdot \mathbf{M}_{\perp Q}^{+}\right) \mathbf{M}_{\perp Q}\right\rangle_{\omega}+\left\langle\mathbf{M}_{\perp Q}^{+}\left(\mathbf{P}_{0} \cdot \mathbf{M}_{\perp Q}\right)\right\rangle_{\omega}-$ $\left\langle\mathbf{M}_{\perp Q} \cdot \mathbf{M}_{\perp Q}^{+}\right\rangle_{\omega} \mathbf{P}_{0}$ et $\left\langle\mathbf{M}_{\perp Q} \cdot \mathbf{M}_{\perp Q}^{+}\right\rangle_{\omega}$ correspondent aux fonctions de corrélation purement magnétiques, non-chirales. Il est facile de vérifier que ces termes induisent une rotation de $180^{\circ}$ du vecteur polarisation incident autour du vecteur d'interaction magnétique $\mathbf{M}_{\mathbf{Q}}$. Le produit croisé $i\left\langle\mathbf{M}_{\perp Q} \times\right.$ $\left.\mathbf{M}_{\perp Q}^{+}\right\rangle_{\omega}$, souvent appelé terme chiral dans la littérature, est associé á la partie antisymétrique des fonctions de corrélation purement magnétiques croisées. Enfin, les termes $\left\langle N_{Q}^{+} \mathbf{M}_{\perp Q}\right\rangle_{\omega}+\left\langle\mathbf{M}_{\perp Q}^{+} N_{Q}\right\rangle_{\omega}$ et $i\left(\left\langle\mathbf{M}_{\perp Q}^{+} N_{Q}\right\rangle_{\omega}-\left\langle N_{Q}^{+} \mathbf{M}_{\perp Q}\right\rangle_{\omega}\right)$, appelés termes d'interférence nucléaire-magnétique (INM), sont respectivement reliés aux parties symétriques et antisymétriques des fonctions de corrélations croisées mixant les composantes nucléaires et les diverses composantes magnétiques. Si elles sont suffisamment fortes, ces dernières peuvent induire, après diffusion, une rotation du vecteur polarisation, donnant ainsi lieu à l'apparition de termes non-diagonaux non nuls dans la matrice de polarisation. Suivant l'usage adopté en diffusion inélastique des neutrons, les différentes composantes vectorielles ( $x, y$ et $z$ ) seront définies par rapport au vecteur de diffusion $\mathbf{Q}$, et les vecteurs repérés par rapport au référentiel de base définie sur la Figure 1. Dans les équations (3.1) et (3.2), la quantité $\left\langle A_{Q} B_{Q}^{+}\right\rangle_{\omega}$ (dite fonction de Van Hove [15]) n'est rien d'autre que la transformée de Fourier de la fonction de corrélation spacio-temporelle $\left\langle A\left(\mathbf{R}_{n}, t\right) B^{+}(\mathbf{0}, 0)\right\rangle$ :

$$
\left\langle A_{Q} B_{Q}^{+}\right\rangle_{\omega}=\frac{1}{2 \pi} \int_{-\infty}^{+\infty} d t e^{i \omega t} \sum_{n}\left\langle A\left(\mathbf{R}_{n}, t\right) B^{+}(\mathbf{0}, 0) e^{i \mathbf{Q} \cdot \mathbf{R}_{n}}\right\rangle
$$

Dans certains cas, il peut s'avérer pratique d'utiliser les susceptibilités généralisées, définies par la relation suivante :

$$
\langle A, B\rangle_{\omega}=i \int_{0}^{+\infty} d t e^{i \omega t}\langle[A(t), B(0)]\rangle=\langle A, B\rangle_{\omega}^{\prime}+i\langle A, B\rangle_{\omega}^{\prime \prime}
$$




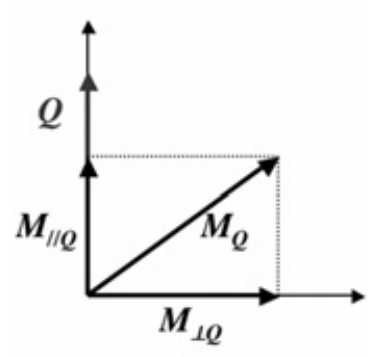

a)

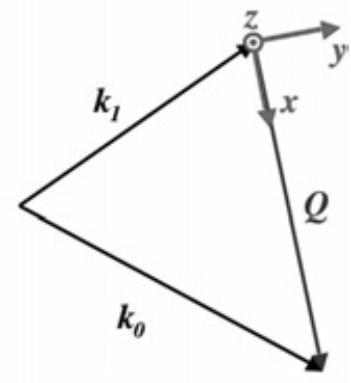

b)

Figure 1. Représentation géométrique de la diffusion magnétique. (a) Règle de sélection des neutrons : seule les composantes de $\mathbf{M}_{\mathbf{Q}}$ perpendiculaires à $\mathbf{Q}$ contribuent au processus de diffusion magnétique. (b) Référentiel de base utilisé en analyse de polarisation : les coordonnées sont définies par rapport au vecteur de diffusion $\mathbf{Q}=\mathbf{k}_{0}-\mathbf{k}_{1}$.

où $\langle A, B\rangle_{\omega}^{\prime}$ représente la partie dispersive et $\langle A, B\rangle_{\omega}^{\prime \prime}$ la partie absorbante. Dans le cas le plus général, la quantité $\langle A, B\rangle_{\omega}^{\prime \prime}$ pourra être complexe. A partir du théorème de fluctuation-dissipation, il est facile d'établir la relation générale suivante entre $\langle A B\rangle_{\omega}$ et $\langle A, B\rangle_{\omega}^{\prime \prime}$ :

$$
\langle A B\rangle_{\omega}=\frac{1}{\pi\left(1-e^{-\hbar \omega / k_{B} T}\right)}\langle A, B\rangle_{\omega}^{\prime \prime}
$$

Les formules s'appliquant au cas élastique peuvent être facilement retrouvées par le remplacement $\frac{k_{1}}{k_{0}} \frac{1}{\pi\left(1-e^{-\hbar \omega / k_{B} T}\right)}\langle A, B\rangle_{\omega}^{\prime \prime} \rightarrow\langle A\rangle\langle B\rangle$, où \langle\rangle traduit la moyenne thermique. Il est facile de montrer que les divers coefficients de la matrice de polarisation dépendent des neuf quantités suivantes :

$$
\begin{aligned}
& N=\left\langle N_{Q} N_{Q}^{+}\right\rangle_{\omega} \\
& M_{y y}=\left\langle M_{Q y} M_{Q y}^{+}\right\rangle_{\omega} \\
& M_{z z}=\left\langle M_{Q z} M_{Q z}^{+}\right\rangle_{\omega} \\
& M_{c h}=i\left(\left\langle M_{Q y} M_{Q z}^{+}\right\rangle_{\omega}-\left\langle M_{Q z} M_{Q y}^{+}\right\rangle_{\omega}\right) \\
& M_{y z}=\left\langle M_{Q y} M_{Q z}^{+}\right\rangle_{\omega}+\left\langle M_{Q z} M_{Q y}^{+}\right\rangle_{\omega} \\
& R_{y}=\left\langle N_{Q} M_{Q y}^{+}\right\rangle_{\omega}+\left\langle N_{Q}^{+} M_{Q y}\right\rangle_{\omega} \\
& I_{y}=i\left(\left\langle N_{Q} M_{Q y}^{+}\right\rangle_{\omega}-\left\langle N_{Q}^{+} M_{Q y}\right\rangle_{\omega}\right) \\
& R_{z}=\left\langle N_{Q} M_{Q z}^{+}\right\rangle_{\omega}+\left\langle N_{Q}^{+} M_{Q z}\right\rangle_{\omega} \\
& I_{z}=i\left(\left\langle N_{Q} M_{Q z}^{+}\right\rangle_{\omega}-\left\langle N_{Q}^{+} M_{Q z}\right\rangle_{\omega}\right)
\end{aligned}
$$

qui sont, dans le cas le plus général, les neuf inconnues du problème que l'on cherche à déterminer. En toute rigueur, des termes supplémentaires dépendant du produit croisé $\mathbf{k}_{0} \times \mathbf{k}_{1}$ devraient apparaître à la fois dans les expressions de la section efficace et de la polarisation lorsque les corrections relativistes [16] et spin-orbite [17] sont prises en compte. De façon intéressante, ces termes introduisent une faible asymétrie droite-gauche dans le processus de diffusion, qui pourrait éventuellement être observée dans des mesures précises. Ces termes, qui contiennent tous le pré-facteur $m_{e} / m_{n}$, rapport de la masse de l'électron à la masse du neutron, sont supposés être prés de trois ordres de grandeur plus petits que les termes inélastiques magnétique-magnétique conventionnels. Ils seront donc complètement négligés par la suite. 
L'expression la plus générale de la section efficace de diffusion inélastique peut être ré-écrite sous la forme linéaire de $\mathbf{P}_{0}$ suivante [11] :

$$
\frac{d^{2} \sigma}{d \Omega} d \omega=\sigma(\mathbf{Q}, \omega)=\sigma_{0}(\mathbf{Q}, \omega)+\mathbf{P}_{0} \sum_{0}(\mathbf{Q}, \omega)
$$

De façon similaire, l'expression de la polarisation peut aussi se mettre sous une forme linéaire :

$$
\mathbf{P} \sigma(\mathbf{Q}, \omega)=T(\mathbf{Q}, \omega) \mathbf{P}_{0}+\sum_{1}(\mathbf{Q}, \omega)
$$

où $\sigma_{0}, \boldsymbol{\Sigma}_{0}$ et $\boldsymbol{\Sigma}_{1}$ peuvent facilement se déduire des équations (3.1) et (3.2). Dans l'équation (3.7), $T$ est un tenseur de rang 2 agissant sur le vecteur $\mathbf{P}_{0}$ selon la règle : $T \mathbf{P}_{0}=T_{\alpha \beta} P_{0 \beta}$. Dans le cas le plus général, $T$ possédera une partie symétrique et une partie antisymétrique :

$$
T_{\alpha \beta}=T_{\alpha \beta}^{S}+\sum_{\gamma} \epsilon_{\alpha \beta \gamma} A_{\gamma},
$$

où $\epsilon_{\alpha \beta \gamma}$ est un pseudo-tenseur unitaire de rang 3 et $\mathbf{A}$ est un vecteur axial, fonction des omposantes du vecteur interaction. L'équation (3.7)) peut être ré-écrite sous la forme condensée suivante :

$$
\mathbf{P} \sigma(\mathbf{Q}, \omega)=T^{S}(\mathbf{Q}, \omega) \mathbf{P}_{0}+\mathbf{A} \times \mathbf{P}_{0}+\sum_{1}(\mathbf{Q}, \omega)
$$

L'équation (3.8) revèle le point très important suivant : les composantes transverses de polarisation ne peuvent provenir que de la partie antisymétrique et résultent nécessairement de l'existence d'un vecteur axial dans le problème. Toute la difficulté du problème en diffusion inélastique sera de trouver un échantillon pour lequel ce vecteur est non nul par symétrie.

Jusqu'à présent, nous avons implicitement supposé que le noyau ne possèdait pas de spin. Le traitement complet du probléme, en tenant compte des effets du spin nucléaire, a ét e ffectué il y a bien longtemps par Blume [8]. Sans entrer plus dans les détails, on peut montrer que les expressions de la polarisation et de la section efficace de diffusion pour le sous-système nucléaire sont très similaires dans leur forme aux équations (3.1) et (3.2), laseule différence étant que, cette fois-ci, les trois composantes du spin nucléaire $I_{\alpha}(\alpha=x, y, z)$ jouent un rôle (il n'y a plus de régle de sélection). Dans la suite, nous ne considérerons que les phénomènes se produisant à des températures situées au dessus de l'échelle du Kelvin. A de telles températures, une bonne approximation est de considérer les spins nucléaires comme totalement décorrélés (de sorte que $\left\langle I_{\alpha}\right\rangle \approx 0$ et $\left\langle I_{\alpha} I_{\beta}\right\rangle \approx \frac{1}{3} I(I+1) \delta_{\alpha \beta}$ ). Les effets du spin nucléaire ne font alors apparaître que les deux termes suivants dans les parties de droites des équations (3.1) et (3.2) $: \frac{k_{1}}{k_{0}}\left[\left\langle\left(\mathbf{P}_{0} \cdot \mathbf{I}_{Q}^{+}\right) \mathbf{I}_{Q}\right\rangle_{\omega}+\left\langle\mathbf{I}_{Q}^{+}\left(\mathbf{P}_{0} \cdot \mathbf{I}_{Q}\right)\right\rangle_{\omega}-\left\langle\mathbf{I}_{Q} \cdot \mathbf{I}_{Q}^{+}\right\rangle_{\omega} \mathbf{P}_{0}\right]$ et $\frac{k_{1}}{k_{0}}\left\langle\mathbf{I}_{Q} \cdot \mathbf{I}_{Q}^{+}\right\rangle_{\omega}$, respectivement. A partir des équations ci-dessus, il est aisé de vérifier que la polarisation additionnelle due à la diffusion incohérente de spin nucléaire ne dépend plus de la direction de la polarisation incidente et pourra donc être éliminée du probléme par différence, comme on le verra ultérieurement. La section efficace correspondante est en outre une fonction $\delta$ de l'énergie $(\propto I(I+1) \delta(\omega))$, qui montre que le processus de diffusion est purement élastique.

\subsection{Polarimétrie neutronique longitudinale}

Théorie

La technique d'Analyse de Polarisation Longitudinale (APL) permet d'accéder expérimentalement à $P_{L}$, la projection de la polarisation finale $\mathbf{P}$ sur la polarisation incidente $\mathbf{P}_{0}$. Dans le cas très réaliste 
pour lequel les termes INM antisymétriques inélastiques peuvent être négligés, les expressions (3.1) et (3.2) se simplifient beaucoup :

$$
\begin{aligned}
\mathbf{P}\left(d^{2} \sigma / d \Omega d \omega\right)=C_{p} & \frac{k_{1}}{k_{0}}\left[\left\langle N_{Q} N_{Q}^{+}\right\rangle_{\omega} \mathbf{P}_{0}\right. \\
& +\left\langle\left(\mathbf{P}_{0} \cdot \mathbf{M}_{\perp Q}^{+}\right) \mathbf{M}_{\perp Q}\right\rangle_{\omega}+\left\langle\mathbf{M}_{\perp Q}^{+}\left(\mathbf{P}_{0} \cdot \mathbf{M}_{\perp Q}\right)\right\rangle_{\omega} \\
& -\left\langle\mathbf{M}_{\perp Q} \cdot \mathbf{M}_{\perp Q}^{+}\right\rangle_{\omega} \mathbf{P}_{0}+i\left\langle\mathbf{M}_{\perp Q} \times \mathbf{M}_{\perp Q}^{+}\right\rangle_{\omega} \\
& \left.+\left\langle N_{Q}^{+} \mathbf{M}_{\perp Q}\right\rangle_{\omega}+\left\langle\mathbf{M}_{\perp Q}^{+} N_{Q}\right\rangle_{\omega}\right] \\
d^{2} \sigma / d \Omega d \omega=C_{\sigma} & \frac{k_{1}}{k_{0}}\left[\left\langle N_{Q} N_{Q}^{+}\right\rangle_{\omega}\right. \\
& +\left\langle\mathbf{M}_{\perp Q} \cdot \mathbf{M}_{\perp Q}^{+}\right\rangle_{\omega}-i \mathbf{P}_{0} \cdot\left\langle\mathbf{M}_{\perp Q} \times \mathbf{M}_{\perp Q}^{+}\right\rangle_{\omega} \\
& +\mathbf{P}_{0} \cdot\left[\left\langle N_{Q}^{+} \mathbf{M}_{\perp Q}\right\rangle_{\omega}+\left\langle\mathbf{M}_{\perp Q}^{+} N_{Q}\right\rangle_{\omega}\right]
\end{aligned}
$$

la composante longitudinale de la polarisation étant donnée par la relation triviale : $P_{L}=\mathbf{P} \cdot \mathbf{P}_{0} / P_{0}$. En plus des termes INM symétriques et du terme chiral, tous susceptibles de créer de la polarisation, l'équation (3.9) contient des termes proportionnels à $\mathbf{P}_{0}$ (qui ne modifient pas la polarisation incidente) et des termes proportionnels à $-\mathbf{P}_{0}$ (qui renversent complètement la polarisation incidente). En utilisant la terminologie usuelle, les premiers seront appelés termes Non Spin-Flip (NSF) et les seconds termes Spin-Flip (SF). En négligeant en première approximation les termes INM symétriques et le terme chiral, il est possible, avec un peu d'algèbre simple, de déduire les expressions des sections efficaces NSF et $\mathrm{SF}$ dans les différents cas où $\mathbf{P}_{0}$ est parallèle à $\mathbf{x}, \mathbf{y}$ et $\mathbf{z}$ :

$$
\begin{aligned}
& \sigma_{x}^{N S F}(\mathbf{Q}, \omega) \propto N \\
& \sigma_{x}^{S F}(\mathbf{Q}, \omega) \propto M_{y y}+M_{z z} \\
& \sigma_{y}^{N S F}(\mathbf{Q}, \omega) \propto N+M_{y y} \\
& \sigma_{y}^{S F}(\mathbf{Q}, \omega) \propto M_{z z} \\
& \sigma_{z}^{N S F}(\mathbf{Q}, \omega) \propto N+M_{z z} \\
& \sigma_{z}^{S F}(\mathbf{Q}, \omega) \propto M_{y y}
\end{aligned}
$$

Les diverses composantes longitudinales de la polarisation en l'absence de termes INM et chiral, sont déduites des sections efficaces NSF et SF à partir de la relation :

$$
P_{\alpha \alpha} \approx \frac{\sigma_{\alpha}^{N S F}-\sigma_{\alpha}^{S F}}{\sigma_{\alpha}^{N S F}+\sigma_{\alpha}^{S F}} P_{0}
$$

Dans les expressions ci-dessus, nous avons une fois de plus omit les termes provenant des spins nucléaires. Compte tenu de la discussion précédente, il est facile de vérifier que la contribution incohérente de spin-nucléaire SF est deux fois plus grande que la contribution NSF [13-18], quelquesoit la direction de $\mathbf{P}_{0}$. On peut voir que la partie cohérente nucléaire (associée par exemple aux phonons ou à d'autres fluctuations de réseau plus complexes) est, elle, toujours NSF. Les contributions magnétiques dues aux électrons non-appariés peuvent être soit SF soit NSF, selon l'orientation relative de $\mathbf{P}_{0}$ et de $\mathbf{Q}$. Les contributions NSF et SF correspondant aux processus de diffusion les plus connus sont résumées dans le Table 1. La situation la plus intéressante est réalisée pour $\mathbf{P}_{0} / / \mathbf{Q}$, car dans ce cas les contributions magnétiques électroniques sont purement SF et les contributions nucléaires purement NSF. En d'autres termes, on a une séparation complète des contributions nucléaires et magnétiques d'origine électronique. Ainsi, la polarimétrie longitudinale sera une technique très utile lorsqu'on cherchera à déterminer indépendamment ou à séparer les contributions magnétiques et structurales. 
Table 1. Proportions des contributions NSF et SF pour quelques processus de diffusion standards.

\begin{tabular}{lcc}
\hline Type de diffusion & $N S F$ & $S F$ \\
\hline Excitations de réseau & 1 & 0 \\
Excitations électroniques magnétiques & $0-1 / 2$ & $1-1 / 2$ \\
Incohérent de spin nucléaire & $1 / 3$ & $2 / 3$ \\
Incohérent isotopique nucléaire & 1 & 0 \\
\hline
\end{tabular}

Une règle simple, nous permettant de déterminer si une contribution magnétique est SF ou NSF, est la suivante :

(i) Les composantes magnétiques parallèles à $\mathbf{P}_{0}$ sont toujours NSF,

(ii) Les composantes magnétiques perpendiculaires à $\mathbf{P}_{0}$ sont toujours $\mathrm{SF}$.

En mesurant les sections efficaces SF ou NSF (le résultat à la fin doit être le même !) pour les trois orientations de la polarisation incidente, $\mathbf{P}_{0}\left\|\mathbf{x}, \mathbf{P}_{0}\right\| \mathbf{y}$ et $\mathbf{P}_{0} \| \mathbf{z}$, il est possible d'accéder indépendamment aux différentes composantes magnétiques du système électronique, pour le même vecteur de diffusion. Cette méthode, connue dans la littérature sous le nom de méthode Champ Vertical - Champ Horizontal (CV-CH), peut aussi bien être utilisée sur des échantillons monocristallins que polycristallins (voir chapitre 5). Dans la mesure $\varnothing^{\prime} u$ les termes INM et le terme chiral peuvent être négligés, les composantes magnétiques selon $\mathbf{y}$ et $\mathbf{z}$ peuvent être déterminées à partir des différences $\sigma_{x}^{S F}-\sigma_{y}^{S F}$ et $\sigma_{x}^{S F}-\sigma_{z}^{S F}$ respectivement, ou de façon équivalente à partir de $\sigma_{y}^{N S F}-\sigma_{x}^{N S F}$ et $\sigma_{z}^{N S F}-\sigma_{x}^{N S F}[19]$. Cette méthode présente l'énorme avantage d'éliminer du problème les contributions nucléaires et le bruit de fond (toutes deux indépendantes de la direction de la polarisation par rapport au vecteur de diffusion $\mathbf{Q}$ ), mais par contre présente le gros défaut d'être très coûteuse en temps de comptage. Dans le cas général où les termes INM symétrique et le terme chiral antisymétrique ne peuvent plus être négligés, les quatres sections efficaces différentielles élémentaires, $\sigma^{++}=\left(d^{2} \sigma / d \Omega d \omega\right)^{++}$, $\sigma^{--}=\left(d^{2} \sigma / d \Omega d \omega\right)^{--}, \sigma^{+-}=\left(d^{2} \sigma / d \Omega d \omega\right)^{+-}$et $\sigma^{-+}=\left(d^{2} \sigma / d \Omega d \omega\right)^{-+}$pour une polarisation initiale appliquée respectivement selon chacune des trois directions cardinales $\mathbf{x}, \mathbf{y}$ ou $\mathbf{z}$, sont données par les relations suivantes :

$$
\begin{aligned}
& \sigma_{x}^{++} \propto N \\
& \sigma_{x}^{--} \propto N \\
& \sigma_{x}^{+-} \propto M_{y y}+M_{z z}-M_{c h} \\
& \sigma_{x}^{-+} \propto M_{y y}+M_{z z}+M_{c h} \\
& \sigma_{y}^{++} \propto N+M_{y y}+R_{y} \\
& \sigma_{y}^{--} \propto N+M_{y y}-R_{y} \\
& \sigma_{y}^{+-} \propto M_{z z} \\
& \sigma_{y}^{-+} \propto M_{z z} \\
& \sigma_{z}^{++} \propto N+M_{z z}+R_{z} \\
& \sigma_{z}^{--} \propto N+M_{z z}-R_{z} \\
& \sigma_{z}^{+-} \propto M_{y y} \\
& \sigma_{z}^{-+} \propto M_{y y}
\end{aligned}
$$

dans lesquelles $M_{c h}$ est le terme chiral et les $R_{\alpha}(\alpha=y, z)$ sont les termes INM symétriques couplant le terme nucléaire et la composante magnétique dans la direction $\alpha$. Maintenant, si le faisceau de neutrons incident n'est pas polarisé (par exemple pour une configuation monochromateur-analyseur graphite-Heusler), les différentes section efficaces pour une polarisation analysée selon les trois directions cardinales $\mathbf{x}, \mathbf{y}$ et $\mathbf{z}$ sont données par les relations :

$$
\sigma_{x}^{0+} \propto N+M_{y y}+M_{z z}+M_{c h}
$$




$$
\begin{aligned}
& \sigma_{x}^{0-} \propto N+M_{y y}+M_{z z}-M_{c h} \\
& \sigma_{y}^{0+} \propto N+M_{y y}+M_{z z}+R_{y} \\
& \sigma_{y}^{0-} \propto N+M_{y y}+M_{z z}-R_{y} \\
& \sigma_{z}^{0+} \propto N+M_{y y}+M_{z z}+R_{z} \\
& \sigma_{z}^{0-} \propto N+M_{y y}+M_{z z}-R_{z}
\end{aligned}
$$

De façon symétrique, en l'absence d'analyse de polarisation (par exemple pour une configuation monochromateuranalyseur Heusler-graphite), on peut aussi définir les sections efficaces de diffusion suivantes :

$$
\begin{aligned}
& \sigma_{x}^{+0} \propto N+M_{y y}+M_{z z}-M_{c h} \\
& \sigma_{x}^{-0} \propto N+M_{y y}+M_{z z}+M_{c h} \\
& \sigma_{y}^{+0} \propto N+M_{y y}+M_{z z}+R_{y} \\
& \sigma_{y}^{-0} \propto N+M_{y y}+M_{z z}-R_{y} \\
& \sigma_{z}^{+0} \propto N+M_{y y}+M_{z z}+R_{z} \\
& \sigma_{z}^{-0} \propto N+M_{y y}+M_{z z}-R_{z}
\end{aligned}
$$

Les six facteurs de structure "longitudinaux" $N, M_{y y}, M_{z z}, M_{c h}, R_{y}$ et $R_{z}$ peuvent être déterminés séparément à partir des relations précédentes, conformement au jeu d'équations suivant :

$$
\begin{aligned}
& N \propto \sigma_{x}^{++}=\sigma_{x}^{--} \\
& M_{y y} \propto \frac{\sigma_{y}^{++}+\sigma_{y}^{--}}{2}-\frac{\sigma_{x}^{++}+\sigma_{x}^{--}}{2}=\frac{\sigma_{x}^{+-}+\sigma_{x}^{-+}}{2}-\frac{\sigma_{y}^{+-}+\sigma_{y}^{-+}}{2} \\
& M_{z z} \propto \frac{\sigma_{z}^{++}+\sigma_{z}^{--}}{2}-\frac{\sigma_{x}^{++}+\sigma_{x}^{--}}{2}=\frac{\sigma_{x}^{+-}+\sigma_{x}^{-+}}{2}-\frac{\sigma_{z}^{+-}+\sigma_{z}^{-+}}{2} \\
& M_{c h} \propto \frac{\sigma_{x}^{-+}-\sigma_{x}^{+-}}{2}=\frac{\sigma_{x}^{0+}-\sigma_{x}^{0-}}{2}=-\frac{\sigma_{x}^{+0}-\sigma_{x}^{-0}}{2} \\
& R_{y} \propto \frac{\sigma_{y}^{++}-\sigma_{y}^{--}}{2}=\frac{\sigma_{y}^{0+}-\sigma_{y}^{0-}}{2}=\frac{\sigma_{y}^{+0}-\sigma_{y}^{-0}}{2} \\
& R_{z} \propto \frac{\sigma_{z}^{++}-\sigma_{z}^{--}}{2}=\frac{\sigma_{z}^{0+}-\sigma_{z}^{0-}}{2}=\frac{\sigma_{z}^{+0}-\sigma_{z}^{-0}}{2}
\end{aligned}
$$

Cette procédure n'est en fait rien d'autre qu'une généralisation de la méthode $\mathrm{CV}$-CH précédemment décrite. Il est important de remarquer ici que les facteurs de structure $M_{y z}, I_{y}$ et $I_{z}$ ne peuvent pas être déterminés du tout par APL. Ils ne sont accessibles que par polarimétrie neutronique sphérique. $M_{c h}, R_{y}$ et $R_{z}$ peuvent aussi être déterminés très précisemment en mesurant la création de polarisation respectivement dans les directions $\mathbf{x}, \mathbf{y}$ ou $\mathbf{z}$, en partant d'un faisceau initialement non polarisé $\left(P_{0}=0\right)$ :

$$
\begin{aligned}
P_{x} & \approx \frac{M_{c h}}{\sigma_{0}} \\
P_{y} & \approx \frac{R_{y}}{\sigma_{0}} \\
P_{z} & \approx \frac{R_{z}}{\sigma_{0}}
\end{aligned}
$$

où $\sigma_{0}=N+M_{y y}+M_{z z}$ n' est rien d' autre que le facteur de structure total pour un faisceau de neutrons totalement dépolarisé. 
Entre autres choses, l'analyse de polarisation longitudinale sera particulièrement utile pour la détermination précise de l'anisotropie des fonctions des corrélations magnétiques dynamiques. Pour un système magnétique quasi isotrope, la polarisation ne dépend que de la direction relative de $\mathbf{Q}$ et $\mathbf{P}_{0}$ :

$$
\mathbf{P} \approx-\frac{\mathbf{Q}\left(\mathbf{Q} \cdot \mathbf{P}_{0}\right)}{Q^{2}}
$$

Cette expression, établie il y a longtemps [1], a été vérifiée expérimentalement pour la premiére fois par diffusion des neutrons polarisés aux petits angles sur un échantillon de nickel [20].

Revenons au problème de la mesure d'une faible contribution nucléaire inélastique superposée à une forte contribution magnétique inélastique, pour un système pour lequel le terme chiral est négligeable $\left(M_{c h} \approx 0\right)$. A partir de l'équation (3.9), on peut établir la relation importante suivante reliant les trois composantes longitudinales de la matrice de polarisation $P_{x x}, P_{y y}$ et $P_{z z}$ et la contribution nucléaire $N$ :

$$
\frac{P_{x x}+P_{y y}+P_{z z}}{P_{0}} \approx-1+\frac{4 N}{N+M_{y y}+M_{z z}}
$$

Bien que beaucoup plus gourmande en temps de comptage que la méthode simple consistant à déterminer la contribution nucléaire directement à partir de la mesure de la contribution NSF pour $\mathbf{P}_{0} \| \mathbf{x}$ (en vertu de la relation $: N \propto \sigma_{x}^{N S F}(\mathbf{Q}, \omega)-B c k^{N S F}$ ), la mesure de $P_{x x}+P_{y y}+P_{z z}$ combinée à l'utilisation de l'équation (3.16) offre l'énorme avantage d'éliminer au premier ordre les effets des composantes magnétiques parasites provenant des spins nucléaires ou électroniques (comme par exemple la diffusion multiple d'une excitation magnétique sur une réflexion de Bragg) et finalement d'être plus précise. Bien évidemment, cette méthode ne marche qu'à la condition de pouvoir déterminer proprement les différents bruits de fond.

\section{Procédure expérimentale}

Comment les composantes longitudinales de la polarisation sont-elles déterminées pratiquement? La Figure 2 montre le principe de la polarimétrie longitudinale sur spectromètre "trois-axes" (TAS). Le sélecteur de polarisation est généralement un système de bobines d'Helmholtz permettant de positionner le vecteur polarisation dans n'importe quelle direction de l'espace. Les différents états $\mid+>$ ou $\mid->$ du spin du neutron peuvent être sélectionnés via un jeu de deux "flippers" situés sur les faisceaux incident et diffusé. La solution complète du problème consiste en la détermination des six facteurs de structure $N, M_{y y}, M_{z z}, M_{c h}, R_{y}$ et $R_{z}$, à partir de la mesure d'un nombre minimal de sections efficaces "non spin-flip" $\sigma_{\alpha}^{++}$et $\sigma_{\alpha}^{--}$, et "spin-flip" $\sigma_{\alpha}^{+-}$et $\sigma_{\alpha}^{-+}(\alpha=x, y$ et $z)$ et en appliquant les relations ci-dessus. En pratique, la procédure consiste à mesurer les quatre intensités $I_{\alpha}^{++}, I_{\alpha}^{+-}, I_{\alpha}^{-+}$et $I_{\alpha}^{--}$(ou au moins une partie d'entre elles), et, éventuellement, les différents bruits de fond associés.

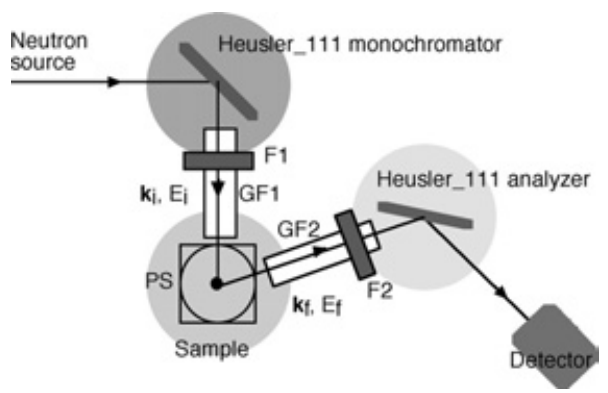

Figure 2. Principe de l'Analyse de Polarisation Longitudinale (APL) sur spectromètre "trois-axes" (GF sont les champs guides ; F sont les "flippers"; PS est le sélecteur de polarisation). 


\subsection{Polarimétrie neutronique sphérique (PNS)}

La détermination expérimentale des termes INM par analyse de polarisation sphérique peut être très intéressante à faire. C'est le cas, par exemple, pour des systèmes où les degrés de liberté magnétiques, de réseau et de charge sont fortement hybridés. Les fonctions de corrélations hybrides résultantes (spin-réseau, orbite-réseau, spin-spin, orbite-orbite, spin-orbite) peuvent alors produire des termes d'interférence non évanescents, qui sont susceptibles de faire tourner légérement le vecteur polarisation, donnant ainsi naissance à des termes non diagonaux de la matrice de polarisation. Comme on l'a vu précédemment, l'observation d'une rotation de la polarisation implique nécessairement l'existence d'au moins un vecteur axial dans le problème. Les bons systèmes prototypes susceptibles de présenter des termes INM (symétriques ou anti symétriques) mesurables sont donc à priori à rechercher parmis les systèmes à électrons fortement corrélés pour lesquels les couplages spin-orbite et/ou spin-réseau sont suffisamment forts, condition nécessaire mais certainement pas suffisante. A notre connaissance, le cas du système décrit par un terme d'interaction vectoriel de type Dzyaloshinskii-Moriya modulé par les excitations de réseau (Dzyaloshinskii-Moriya dynamique) est le seul à avoir été traité théoriquement [9,64]. Quoiqu'il en soit, en plus des trois composantes longitudinales $x x, y y$ et $z z$ usuelles, la PNS permet aussi de déterminer les composantes transverses $x y, y x, x z, z x, y z$ et $z y$, inaccessibles par les autres techniques. Dans la section suivante, nous allons donner les principales relations permettant de relier les différentes composantes de polarisation $P_{\alpha \beta}$ et les divers termes d'interférence.

\section{Relations de base de la PNS inélastique}

Pour la suite, il sera utile de ré-introduire les quantités suivantes :

$$
R_{\alpha}=\left\langle M_{Q}^{\alpha+} N_{Q}\right\rangle_{\omega}+\left\langle M_{Q}^{\alpha} N_{Q}^{+}\right\rangle_{\omega}
$$

définie précédemment et caractérisant la partie symétrique des termes INM mettant en jeu les composantes magnétiques selon la direction $\alpha$.

$$
I_{\alpha}=i\left[\left\langle M_{Q}^{\alpha+} N_{Q}\right\rangle_{\omega}-\left\langle M_{Q}^{\alpha} N_{Q}^{+}\right\rangle_{\omega}\right]
$$

qui caractérise la partie antisymétrique des termes INM, et

$$
\sigma_{0}=\left\langle N_{Q} N_{Q}^{+}\right\rangle_{\omega}+\left\langle M_{Q}^{y} M_{Q}^{y+}\right\rangle_{\omega}+\left\langle M_{Q}^{z} M_{Q}^{z+}\right\rangle_{\omega}
$$

qui n'est rien d'autre que la section efficace totale pour un faisceau incident non polarisé, en négligeant les termes INM et chiral, tous supposés être beaucoup plus petits que les contributions magnétiques usuelles. De même que le terme chiral $M_{c h}=i\left(\left\langle M_{Q}^{y} M_{Q}^{z+}\right\rangle_{\omega}-\left\langle M_{Q}^{z} M_{Q}^{y+}\right\rangle_{\omega}\right)$, les termes INM symétriques peuvent conduire à polariser un faisceau de neutrons initialement non polarisé. Les diverses composantes du vecteur polarisation résultant sont données par les relations ci-dessous :

$$
\begin{aligned}
P_{x} & \approx \frac{M_{c h}}{\sigma_{0}} \\
P_{y} & \approx \frac{R_{y}}{\sigma_{0}} \\
P_{z} & \approx \frac{R_{z}}{\sigma_{0}}
\end{aligned}
$$

Ainsi, la mesure des trois composantes du vecteur polarisation après diffusion doit en principe permettre de déterminer le terme chiral et les parties symétriques des termes INM. Malgré tout, la détermination des parties antisymétriques des termes INM requérira nécessairement la mesure par PNS d'un nombre minimal de composantes non diagonales de la matrice de polarisation. A partir des équations générales 
précédentes, et en supposant les termes INM et chiral inélastiques petits (mais non nuls!), il est facile d'en déduire les relations suivantes donnant les composantes transverses $x y, y x, x z, z x, y z$ et $z y$ (au premier ordre) :

$$
\begin{aligned}
P_{x y} & \approx \frac{R_{y}}{\sigma_{0}}-\frac{I_{z}}{\sigma_{0}} P_{0} \\
P_{y x} & \approx \frac{M_{c h}}{\sigma_{0}}+\frac{I_{z}}{\sigma_{0}} P_{0} \\
P_{x z} & \approx \frac{R_{z}}{\sigma_{0}}+\frac{I_{y}}{\sigma_{0}} P_{0} \\
P_{z x} & \approx \frac{M_{c h}}{\sigma_{0}}-\frac{I_{y}}{\sigma_{0}} P_{0} \\
P_{y z} & \approx \frac{R_{z}}{\sigma_{0}}+\frac{M_{y z}}{\sigma_{0}} P_{0} \\
P_{z y} & \approx \frac{R_{y}}{\sigma_{0}}+\frac{M_{y z}}{\sigma_{0}} P_{0}
\end{aligned}
$$

Le terme symétrique purement magnétique $M_{y z}=\left\langle M_{Q}^{y} M_{Q}^{z+}\right\rangle_{\omega}+\left\langle M_{Q}^{z} M_{Q}^{y+}\right\rangle_{\omega}$ met en jeu des fonctions de corrélation couplant les composantes magnétiques selon $\mathbf{y}$ et $\mathbf{z}$, comme le fait aussi le terme chiral antisymétrique. Il est intéressant de noter que, dans le cas général, la matrice de polarisation $P_{\alpha \beta}$ $(\alpha, \beta=x, y, z)$ n'est pas une matrice symétrique (par exemple $P_{x y}$ peut être différent de $P_{y x}$ ). Les différents facteurs de structure mis en jeu peuvent être déterminés séparément à partir des combinaisons symétriques ou antisymétriques appropriées des composantes transverses mesurées avec une polarisation incidente $\mathbf{P}_{0}$ et renversée $\left(-\mathbf{P}_{0}\right)$, conformément aux relations :

$$
\begin{aligned}
& M_{c h} \approx \frac{P_{y x}+P_{\bar{y} x}}{2} \sigma_{0} \approx \frac{P_{z x}+P_{\bar{z} x}}{2} \sigma_{0} \\
& M_{y z} \approx \frac{P_{y z}-P_{\bar{y} z}}{2 P_{0}} \sigma_{0} \approx \frac{P_{z y}-P_{\bar{z} y}}{2 P_{0}} \sigma_{0} \\
& R_{y} \approx \frac{P_{x y}+P_{\bar{x} y}}{2} \sigma_{0} \approx \frac{P_{z y}+P_{\bar{z} y}}{2} \sigma_{0} \\
& I_{y} \approx \frac{P_{x z}-P_{\bar{x} z}}{2 P_{0}} \sigma_{0} \approx-\frac{P_{z x}-P_{\bar{z} x}}{2 P_{0}} \sigma_{0} \\
& R_{z} \approx \frac{P_{x z}+P_{\bar{x} z}}{2} \sigma_{0} \approx \frac{P_{y z}+P_{\bar{y} z}}{2} \sigma_{0} \\
& I_{z} \approx \frac{P_{x y}-P_{\bar{x} y}}{2 P_{0}} \sigma_{0} \approx-\frac{P_{y x}-P_{\bar{y} x}}{2 P_{0}} \sigma_{0}
\end{aligned}
$$

On notera que la procédure utilisée pour la détermination expérimentale de $N, M_{y y}$ et $M_{z z}$ en PNS est exactement la même qu'en APL, puisqu'elle n'est basée que sur l'utilisation des termes longitudinaux de la matrice de polarisation. Pour un signal purement magnétique (c'est à dire en négligeant tous les termes impliquant l'opérateur nucléaire $\mathrm{N}$ ), on peut déduire pour les composantes longitudinales et transverses les relations suivantes :

$$
P_{x x} \approx-\frac{\sigma_{M} P_{0}-M_{c h}}{\sigma_{M}-M_{c h} P_{0}}
$$




$$
\begin{aligned}
& P_{y y} \approx-P_{z z} \approx \frac{M_{y y}-M_{z z}}{\sigma_{M}} P_{0} \\
& P_{x y} \approx P_{x z} \approx 0 \\
& P_{y x} \approx P_{z x} \approx \frac{M_{c h}}{\sigma_{M}} \\
& P_{y z} \approx P_{z y} \approx \frac{M_{y z}}{\sigma_{M}} P_{0}
\end{aligned}
$$

expressions dans lesquelles $\sigma_{M}=M_{y y}+M_{z z}$ est le facteur de structure magnétique total. On peut ainsi prévoir l'existence de composantes non diagonales $P_{y x}$ et $P_{z x}$ finies pour un système présentant des fonctions de corrélations croisées $\left\langle y z>\right.$ finies (termes $M_{c h}$ et $M_{y z}$ inélastiques éventuellement non nuls).

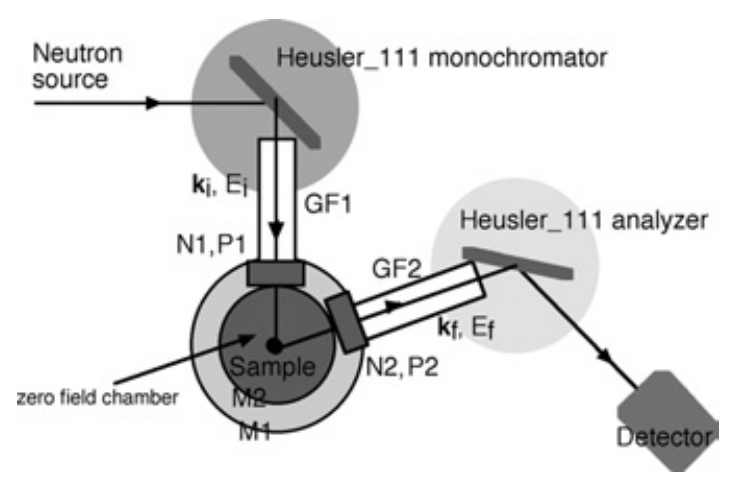

Figure 3. Principe de la polarimétrie neutronique sphérique (PNS) sur spectromètre "trois-axes" et avec CRYOPAD (GF sont les champs guides ; $\mathrm{N}$ et $\mathrm{P}$ sont respectivement les bobines de nutations et de précessions ; $\mathrm{M} 1$ et M2 sont deux écrans Meissner supraconducteurs).

\section{Procédure expérimentale}

Le seul dispositif actuellement capable (en 2005!) de mesurer avec précision les termes non diagonaux de la matrice de polarisation est CRYOPAD (acronyme de Cryogenic Polarisation Analysis Device). Le principe de ce dispositif est décrit dans les références [26,27]. Le schéma de principe de la PSN sur spectromètre trois-axes est donné sur la Figure 3. La procédure expérimentale consiste à déterminer pour chaque configuration incidente $\alpha$ et $\bar{\alpha}\left(\alpha=\mathrm{x}, \mathrm{y}\right.$ ou z), les trois composantes $P_{f \beta}(\alpha)(\beta=\mathrm{x}, \mathrm{y}, \mathrm{z})$ en mesurant les intensités corrigées $d u$ bruit de fond $I_{\alpha \beta}^{+}$et $I_{\alpha \beta}^{-}$respectivement, associées aux états de spin $|+\rangle$ et $|-\rangle$ (18 contributions en tout), selon la relation :

$$
P_{\beta}(\alpha)=\frac{I_{\alpha \beta}^{+}-I_{\alpha \beta}^{-}}{I_{\alpha \beta}^{+}+I_{\alpha \beta}^{-}}
$$

Les différents facteurs de structure sont déduits des combinaisons linéaires symétriques ou antisymétriques appropriées, en appliquant le jeu de relations précédent. Sans aucun doute, la difficulté majeure de la méthode en diffusion inélastique des neutrons, réside dans la faiblesse des effets attendus, qui implique dans la plupart des cas des temps de comptages relativement longs, nécessaires à l'obtention de la précision statistique requise (plusieurs heures par point dans le cas des composés supraconducteurs Ĺ haute température critique!). 


\section{APPLICATION DE L'ANALYSE DE POLARISATION DES NEUTRONS EN DIFFUSION INÉLASTIQUE}

Dans cette section, nous allons décrire un certain nombre d'exemples caractéristiques montrant l'utilité et la puissance de la polarimétrie neutronique inélastique pour l'étude des excitations magnétiques et structurales. Comme nous l'avons vu précédemment, l'analyse de polarisation longitudinale est une méthode incontournable pour la séparation des diverses composantes magnétiques ou pour la détermination indépendante des contributions structurales et magnétiques. Nous allons illustrer notre propos à partir de plusieurs exemples pris dans le domaine du magnétisme des systèmes de spins quantiques à basse dimensionalité ou des matériaux de terres-rares anormales, de la supraconductivité haute- $\mathrm{T}_{c}$, et bien d'autres. L'utilité de la polarimétrie neutronique sphérique sera discutée à partir de l'étude pionnière du composé spin-Peierls $\mathrm{CuGeO}_{3}$.

\subsection{Nature des plans non-magnétiques dans $\mathrm{CeSb}$}

Parmi les composés du cérium, le monopnictide CeSb, qui cristallise dans la structure cubique facecentrées, présente des propriétés magnétiques assez remarquables et uniques [28]. Ce matériau se caractérise en particuliers par une anisotropie axiale des moments magnétiques très forte selon les directions d'ordre quatre $<001>$ [29] et un diagramme de phase H-T très complexe [30], mettant en scéne au moins seize phases différentes en dessous de $T_{N} \approx 16.5 \mathrm{~K}$, selon les échantillons. Toutes ces phases

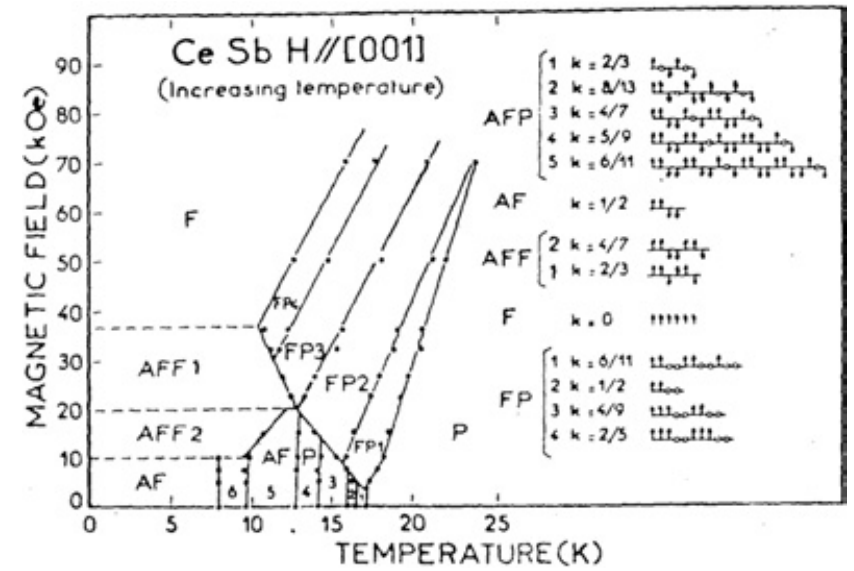

Figure 4. Diagramme de phase $\mathrm{H}-\mathrm{T}$ de $\mathrm{CeSb}$.

sont associées à des vecteurs d'onde commensurables $\mathbf{k}=[00 k]$ et peuvent être décrites comme un empilement de plans (001) ferromagnétiques (F) et/ou non-magnétiques (P). Par exemple, la phase AFF2, décrite par la séquence ++--++- (associée à une composante de vecteur d'onde $k=4 / 7$ ), ne possède pas de plans non-magnétiques, alors que la phase FP2, qui est décrite par la séquence ++00 (associée à $k=1 / 2$ ) possède autant de plans $\mathrm{F}$ que de plans $\mathrm{P}$. Un problème clé à résoudre dans $\mathrm{CeSb}$ concernait la compréhension de la nature des plans non-magnétiques [32]. Sont-ils paramagnétiques (c'est à dire associés à une aimantation thermique moyenne nulle comme dans un état paramagnétique classique) ou reflètent-ils l'existence d'un état fondamental singulet non-magnétique de type Kondo [31]? La technique d'analyse de polarisation longitudinale (APL), qui permet, comme on l'a vu plus 
haut, de sonder indépendamment la nature SF et NSF de la réponse magnétique inélastique, a pu apporter des informations cruciales pouvant aider à répondre à cette question. Pour un champ magnétique appliqué selon la direction verticale [001] (perpendiculaire au plan de diffusion $(\mathbf{a}, \mathbf{b})$ ), une excitation paramagnétique de type champ cristallin devrait être à la fois SF et NSF, avec $I_{S F} \approx I_{N S F}$, alors que toute excitation singulet-triplet devrait apparaître comme principalement $\operatorname{SF}\left(I_{N S F} \approx 0\right)$. De plus il est connu que, pour des moments magnétiques ordonnés pointant selon la direction [001], les ondes de spin (excitations transverses) associées aux plans $\mathrm{F}$ ne devraient être visible que dans le canal SF. Des expériences de diffusion inélastique de neutrons polarisés ont été réalisées dans la phase AFF2 $(H=18 \mathrm{kG} ; T=6 \mathrm{~K})$, qui ne contient pas de plans $\mathrm{P}$, et dans la phase FP2 $(H=18$ $\mathrm{kG} ; T=15 \mathrm{~K}$ ), qui contient pour moitié de plans $\mathrm{P}$. La Figure 5 montre des scans typiques à $\mathrm{Q}$ constant réalisés dans la phase $\mathrm{AFF} 2$ pour les deux vecteurs de diffusion $\mathbf{Q}=(0,-1.1,0)$, situé à proximité du bord de zone de Brillouin, et $\mathbf{Q}=(0,-2.2,0)$, situé à proximité du centre de zone de Brillouin. Comme attendu pour des excitations purement tranverses, les intensités diffusées sont principalement SF alors que l'énergie du mode (respectivement 3 et $4.4 \mathrm{meV}$ ) suit la courbe de dispersion en champ nul, légéremment décalée de la valeur de l'énergie Zeeman $m_{0} H \approx 0.22 \mathrm{meV}$ attendure pour un champ appliqué de $18 \mathrm{kG}$. Des measures similaires ont été réalisées dans la phase FP2 contenant autant de plans $\mathrm{P}$ que de plans F. La Figure 6 résume les résulats principaux qui ont été obtenus. Pour le vecteur de diffusion $\mathbf{Q}=(0,-2.2,0)$, associé à un vecteur d'onde situé à proximité du centre de zone, deux modes distincts ont été observés dans le canal SF à des énergies de $3.45 \mathrm{meV}$ et $4.5 \mathrm{meV}$, tandis que dans le canal NSF, seul un mode est observé aux alentours de $3.45 \mathrm{meV}$. Par comparaison avec les données précédentes obtenues dans la phase AFF2, le mode à $4.5 \mathrm{meV}$ est attribué à une excitation dans les plans $\mathrm{F}$. Le mode à $3.45 \mathrm{meV}$, à la fois SF et NSF (avec $I_{S F} \approx I_{N S F}$ ), est attribué à une excitation dans les plans $\mathrm{P}$. Les résultats en $\mathbf{Q}=(0,-1.1,0)$, bien que plus difficiles à analyser étant donné le fort recouvrement des deux modes, confirment qualitativement la conclusion ci-dessus. Ainsi, les plans $\mathrm{P}$ mis en évidence à basse température sont de vrais plans paramagnétiques.

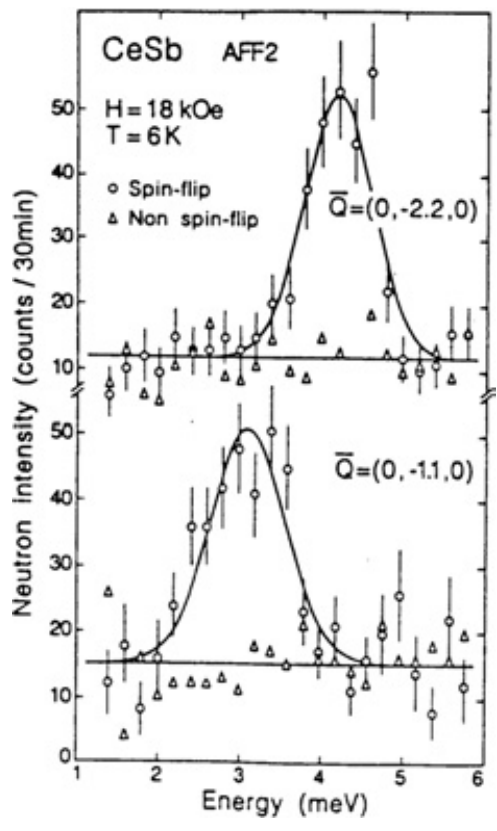

Figure 5. Excitations en centre et bord de zone dans la phase AFF2 de CeSb. 

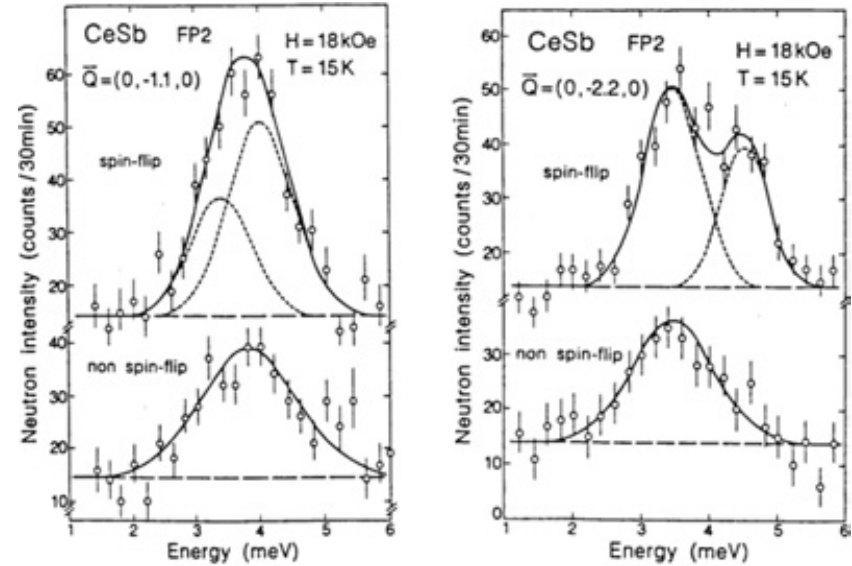

Figure 6. Excitations en centre et bord de zone dans la phase FP2 de CeSb.

Les excitations associées sont en tout point similaires aux excitations de champ cristallin observées dans la phase paramagnétique de CeSb, au dessus de $T_{N}$. L'analyse quantitative de la forme de la réponse inélastique montre que les largeurs en énergie des modes associés aux plans $\mathrm{P}$ (en particulier leurs composantes NSF) sont beaucoup plus grandes que celles des modes associés aux plans F, ce qui implique pour les premiers des temps de vie plus courts, un fait bien compris à partir du désordre plus fort qui est censé exister dans les plans $\mathrm{P}$.

\subsection{Excitations de type solitons dans le composé antiferromagnétique classique en chaîne TMMC}

Dans cette sous-section, nous allons illustrer sur un exemple simple combien utile peut être l'analyse de polarisation longitudinale pour la détermination des dépendances en vecteur d'onde et en énergie des différentes composantes magnétiques entrant en jeu dans un matériau magnétique donné. Dans le système classique antiferromagnétique quasi uni-dimensionnel (1D) de spins de Heisenberg soumis à un champ magnétique appliqué perpendiculairement à l'axe des chaînes, il a été établi il y a longtemps que, en plus des excitations linéaires habituelles de type ondes de spins (associées à des oscillations de spin de faibles amplitudes), des excitations non-linéaires, appelées solitons, jouaient un rôle fondamental dans le comportement thermique [33,34]. Ces excitations, qui sont associées à des rotations de spin de grande amplitude ( $\pi$ ou $2 \pi$ ), peuvent être vues comme des parois de domaines mobiles le long de la chaîne, séparant deux configurations différentes de l'état fondamental dégénéré. Dans un système quasi1D pour lequel les couplages inter-chaînes peuvent être négligés, de telles parois de domaines peuvent facilement se déplacer le long de la chaîne, donnant lieu à une dynamique de spin très caractéristique. Dans le cas antiferromagnétique, la solution qui minimise l'énergie libre correspond à une rotation de $\pi$ de chacun des deux sous-réseaux, comme illustré sur la Figure 7. Le déplacement des solitons entraîne un retournement des spins (un "flip") qui doit clairement se manifester dans les facteurs de structure dynamiques. Selon la théorie [33,34], l'énergie du soliton est directement reliée à la valeur du champ magnétique appliqué, $H$, par la relation linéaire : $E_{S}=g \mu_{B} S H$. La distance moyenne entre deux solitons adjacents définie la longueur de corrélation du système, $\xi$, reliée à la densité de solitons $n_{S}$ par la relation : $\xi^{-1}=4 n_{S}$. Comme on peut facilement le vérifier sur la Figure 7, les solitons présentent à la fois des composantes de spin parallèles et perpendiculaires à la direction du champ appliqué. En particulier, les spins associés aux solitons contribuent principalement au facteur de structure $S_{\|}(q, \omega)$, 


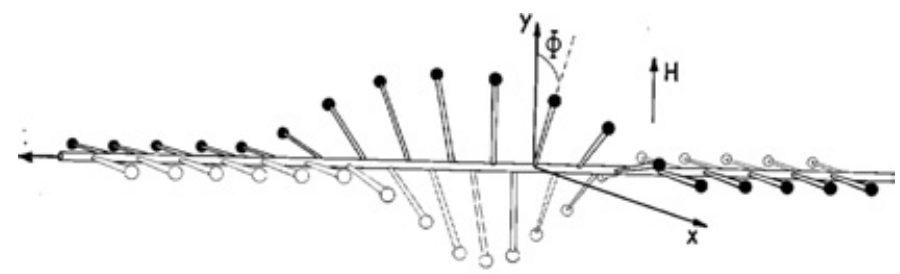

Figure 7. Structure du soliton antiferromagnétique. Le soliton n'est rien d'autre qu'une paroi de domaine se déplaçant selon l'axe de la chaîne.

alors que $S_{\perp}(q, \omega)$ reflète essentiellement le comportement des spins entre solitons. Des expressions analytiques pour $S_{\perp}(q, \omega)$ et $S_{\|}(q, \omega)$ ont été dérivées par Mikeska [33] et Maki [34] dans le cadre du modèle du gaz de solitons sans interaction. Les contributions sont toutes les deux de type quasi-élastique, centrées autour de $q \approx \pi$ et $\omega \approx 0$, et sont données par les relations suivantes :

$$
S_{\perp}(q, \omega) \propto \frac{\Gamma_{\omega} / \Gamma_{q}}{\left[\Gamma_{\omega}^{2}+\left(1+\tilde{q}^{2} / \Gamma_{q}^{2}\right)+\omega^{2}\right]^{3 / 2}}
$$

dans lesquelles les largeurs caractéristiques $\Gamma_{q} \approx 4 n_{S}$ et $\Gamma_{\omega} \approx 4 n_{S} v_{0}$; et où $v_{0} \approx c_{0} \sqrt{\frac{2 k_{B} T}{\pi E_{S}}}$ est la vitesse thermique quadratique moyenne des solitons, reliée à la vitesse d'onde de spin $c_{0} \approx 2 J a$ et l'énergie du soliton $E_{S}$. Et,

$$
S_{\|}(q, \omega) \propto \frac{n_{S} e^{-\left(\omega / v_{0} \tilde{q}\right)^{2}}}{v_{0} \tilde{q} \cosh ^{2}(\pi \tilde{q} / 2 m)}
$$

où $\tilde{q}=q-\pi ; n_{S} \approx \sqrt{\frac{2}{\pi}} \frac{E_{S}}{J S^{2}} \sqrt{\frac{E_{S}}{k_{B} T}} e^{-\frac{E_{S}}{k_{B} T}}$ et $m=\frac{E_{S}}{2 J S^{2} a}$ est la masse du soliton. Dans les équations (4.1) et (4.2), $a$ et $J$ sont respectivement la distance spin-spin et la constante de couplage entre spins adjacents. L'allure des dépendances en $q$ et $\omega$ des facteurs de structure théoriques $S_{\perp}(q, \omega)$ et $S_{\|}(q, \omega)$ est donnée sur les Figures $8 \mathrm{a}\left(S_{\|}\right)$et $8 \mathrm{~b}\left(S_{\perp}\right)$. Pour des conditions d'observation usuelles, $T \sim 2$ à $5 \mathrm{~K}$ et $H \sim 2$ à 5 T, l'extension spatiale du soliton est au moins un ordre de grandeur plus petite que la distance moyenne soliton-soliton, ce qui implique que $S_{\|}(\pi, 0) \ll S_{\perp}(\pi, 0)$. En conséquence, il sera quasiment
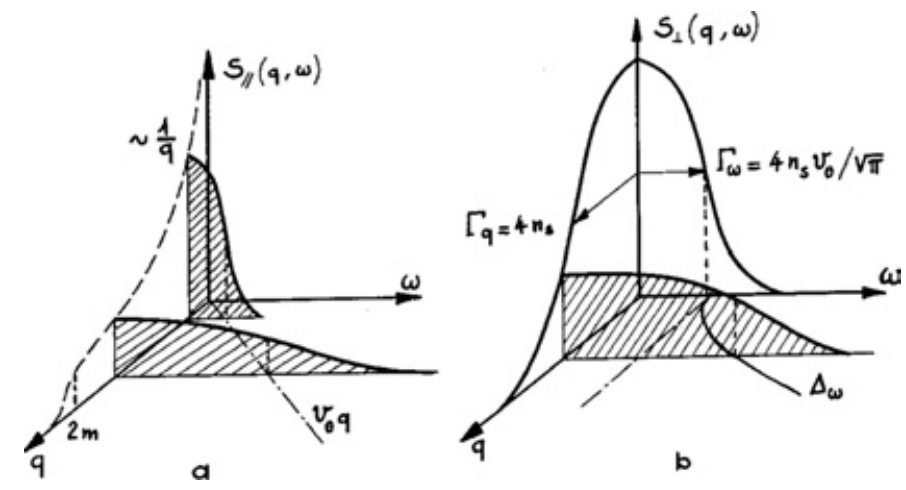

Figure 8. Facteurs de structure théoriques $S_{\|}(q, \omega)$ (a) et $S_{\perp}(q, \omega)$ (b). Tous deux donnent naissance à des contributions quasi-élastiques dépendant fortement de $T$ et $H$. Les différentes courbes donnent une idée de la forme des dépendances en énergie pour différents vecteurs d'onde. 


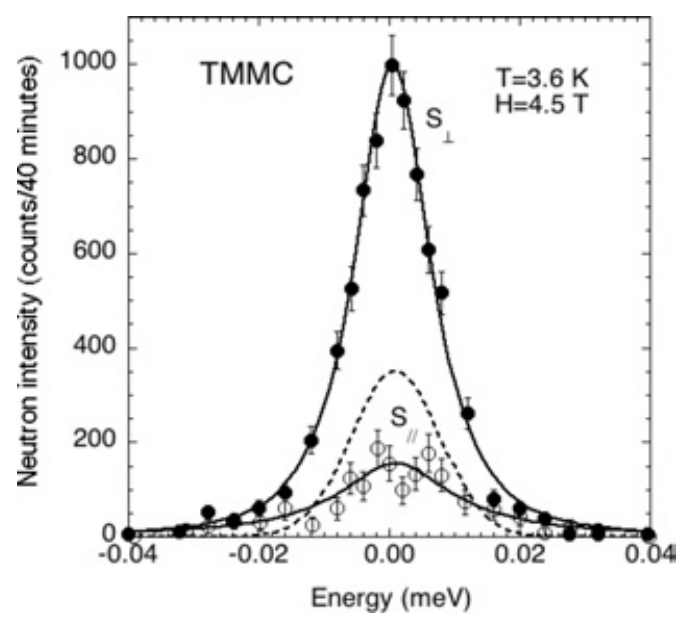

Figure 9. Spectres en fréquence des fluctuations magnétiques observés dans les directions parallèles et perpendiculaires au champ magnétique appliqué ( $\circ$ et $\bullet$, respectivement).

impossible de déterminer précisemment $S_{\|}(q, \omega)$ par diffusion des neutrons non-polarisés [35]. Au contraire, la polarimétrie longitudinale avec $\mathbf{P}_{0} \| \mathbf{H}$ est particulièrement bien adaptée puisque $S_{\|}(q, \omega)$, associée aux composantes de spin parallèles au champ, est entièrement NSF, alors que $S_{\perp}(q, \omega)$, associée aux composantes de spin perpendiculaires au champ, est entièrement SF. Un exemple de résultat expérimental est montré sur la Figure 9 pour une température $T=3.6 \mathrm{~K}$ et un champ magnétique appliqué $H=4.5 \mathrm{~T}$. Le détail des conditions expérimentales ansi que l'ensemble des résultat obtenus sont décrit dans la référence [36]. Pour $S_{\perp}$, la situation expérimentale peut être reproduite à partir de l'équation (4.1) décrivant le mode de flipping dans le modèle du gaz de solitons sans interaction. Cependant, comme le montre la courbe en traits pointillés de la Figure 9, ce modèle un peu trop simpliste est incapable de reproduire quantitativement les données expérimentales pour $S_{\|}$. Un bien meilleur accord (courbe en traits continue de la Figure 9) peut être obtenu en tenant compte de l'amortissement dynamique du mode de soliton, provenant essentiellement des interactions soliton-magnon et solitonsoliton [37] et d'effets additionnels de renormalisation thermique [36]. A notre connaissance, l'APL est la seule technique permettant d'étudier directement et séparement les dépendances en champ et en température des différentes contributions solitoniques.

4.3 Séparation des contributions magnétiques et structurales dans le supraconducteur $\mathrm{L}$ haute température critique $\mathrm{YBa}_{2} \mathrm{Cu}_{3} \mathrm{O}_{6+x}$

Un interêt supplémentaire et important de l'APL est qu'elle permet la séparation des contributions structurales et magnétiques, qui peuvent apparaître superposées sur une partie plus ou moins large de l'espace $\mathbf{q}-\omega$. C'est en particulier le cas pour le cuprate supraconducteur à haute- $\mathrm{T}_{c} \mathrm{YBa}_{2} \mathrm{Cu}_{3} \mathrm{O}_{6+x}(\mathrm{YBCO})$. Une caractéristique générique des supraconducteurs à haute- $\mathrm{T}_{c}$ est qu'ils contiennent tous des plans $\mathrm{CuO}_{2}$, qui forment dans les composés parents non-dopés un système isolant quasi-2D antiferromagnétique ordonné à grande distance [38-41]. Cette constatation pourait en particuliers signifier que le mécanisme de couplage des porteurs de charge responsable de la supraconductivité dans ces matériaux se fait par l'intermédiaire des fluctuations magnétiques, qui, effectivement, subissent de profondes modifications lors du dopage et de la transition métal-isolant associée [41-42]. Même si elle n'apparaîssent pas comme directement responsables du mécanisme d'appariement, les fluctuations magnétiques permettent au moins de sonder microscopiquement la nature du phénomène. Ainsi, un des points clé pour la 
compréhension du mécanisme d'appariement des porteurs de charge dans les cuprates à haute- $\mathrm{T}_{c}$ concerne la détermination précise des dépendances en fonction de la température et du taux de dopage des spectres d'excitations magnétiques et de réseau. Dans YBCO, ce problème a été étudié au début des années 90, à partir d'expériences très détaillées de diffusion inélastique de neutrons non-polarisés [39,41]. Dans la plupart des études, la difficulté était d'extraire proprement les contributions magnétiques relevantes des contributions nucléaires (comme les contributions parasites de Bragg, la diffusion multiple, les contributions des phonons, etc ... ) et autres contributions parasites. Le problème qui se pose est bien illustré sur la Figure 10, qui montre un scan typique à Q constant obtenu en diffusion des neutrons non-polarisés sur le composé légèrement sur-dopé $\mathrm{YBa}_{2} \mathrm{Cu}_{3} \mathrm{O}_{6.92}$, supraconducteur en dessous de $92 \mathrm{~K}$.

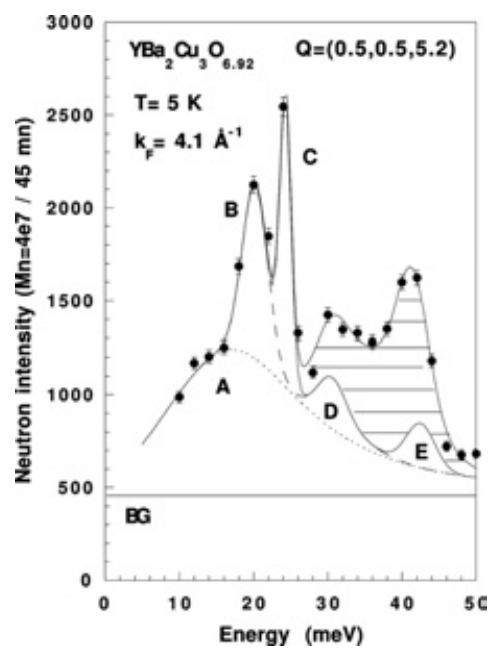

Figure 10. Scan typique à $\mathrm{Q}$ constant obtenu en neutrons non-polarisés en $\mathbf{Q}=(0.5,0.5,5.2)$ dans la phase supraconductrice de $\mathrm{YBa}_{2} \mathrm{Cu}_{3} \mathrm{O}_{6.92}$, montrant les différentes contributions à la diffusion $(\mathrm{A}, \mathrm{B}, \mathrm{D}$ et $\mathrm{E}$ : contributions structurales inélastiques ; C : contamination de Bragg ; BG : bruit de fond ; Zone hachurée : signal magnétique).

Dans ce cas d'école, la contribution magnétique (zone hachurée) peut être au moins partiellement estimée à partir de l'analyse "différentielle" de la dépendance en température de la diffusion inélastique. La méthode APL, à partir de la mesure des contributions SF et NSF dans la configuration $\mathbf{P}_{0} \| \mathbf{Q}$, peut apporter une solution plus simple, plus complète et finalement plus précise au problème. Comme on l'a vu précédemment, dans a configuration $\mathbf{P}_{0} \| \mathbf{Q}$ les excitations de réseau ne contribuent qu'au canal NSF, alors que les contributions magnétiques ne doivent contribuer qu'au canal SF, autorisant ainsi la séparation quasi-parfaite des degrés de liberté structuraux et magnétiques. Un résultat caractéristique obtenu en APL est présenté sur la Figure 11, qui montre un résultat de scan à Q constant réalisé au centre de zone antiferromagnétique $\mathbf{Q}=(1.5,0.5,1.7)$ en NSF et $\mathrm{SF}$ dans le composé $\mathrm{YBa}_{2} \mathrm{Cu}_{3} \mathrm{O}_{6.85}$ $\left(T_{c} \approx 89 \mathrm{~K}\right)$. Ces scans permettent de déterminer séparément les contributions de réseau et les contributions magnétiques $[43,44]$. Comme il est maintenant bien admis, la principale caractéristique du spectre d'excitations magnétiques dans $\mathrm{YBa}_{2} \mathrm{Cu}_{3} \mathrm{O}_{6+x}$ pour $x$ proche du dopage optimum $\left(x_{\text {opt }} \approx 0.95\right.$ pour le système YBCO) est la présence d'un mode résonant centré à $(\pi, \pi)$, dont le maximum d'intensité est observé à l'énergie caractéristique $E_{R} \approx 41 \mathrm{meV}$, et qui disparait quasiment à $\mathrm{T}_{c}$. A la précision des expériences de neutrons polarisés, on voit que la réponse magnétique pour $x \approx 0.85$ n'est mesurable que dans la gamme d'énergie $25-50 \mathrm{meV}$. De façon remarquable, ce pic résonant semble être une entité magnétique caractéristique de l'état supraconducteur, qui disparait presque complètement dans l'état normal [41]. 


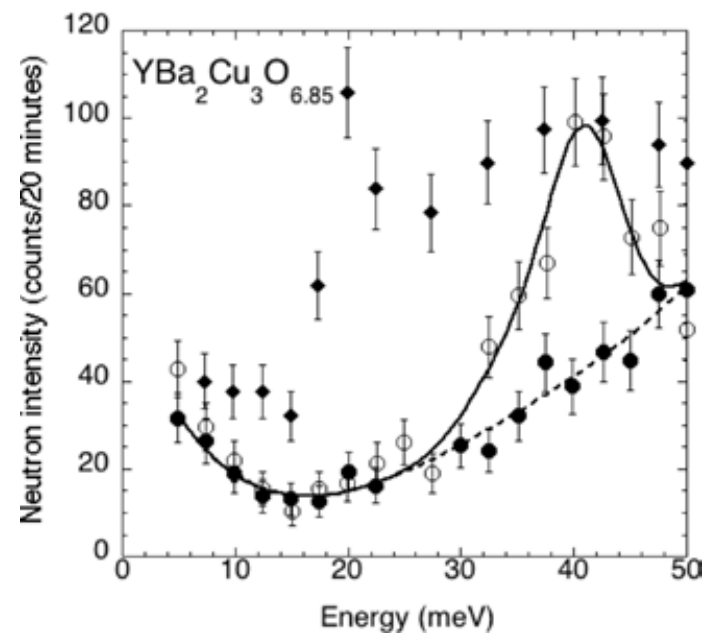

Figure 11. Scan à Q constant dans les canaux "spin-flip" (o) et "non spin-flip" ( $)$ au centre de zone antiferromagnétique en $\mathbf{Q}=(1.5,0.5,1.7)$ et sur le bruit de fond en $\mathbf{Q}=(1.8,0.6,1.7)(\bullet)$. Ces mesures ont été réalisées dans la configuration $\mathbf{P}_{0} \| \mathbf{Q}$, permettant une séparation nette des contributions magnétiques et structurales.

\subsection{Spectre d'excitation magnétique du composé spin-Peierls $S=1 / 2 \mathrm{CuGeO}_{3}$}

Le germanate $\mathrm{CuGeO}_{3}$ est considéré dans la littérature comme un très bon prototype de système spinPeierls [45-47,49]. En dessous d'une température caractéristique $T_{S P} \approx 14 \mathrm{~K}$ (dite température de transition spin-Peierls), les susceptibilités magnétiques selon les trois directions cristallographiques principales chutent exponentiellement à zéro [45], tandis que simultanément une distorsion (en fait une dimérisation décrite par le vecteur d'onde structural $k_{S P}=(1 / 2,0,1 / 2)$ ) se développe à basse température [48]. Dans ce matériau, de forts couplages spin-réseau sont présents, qui sont responsables de la dimérisation en question et à la fois de l'apparition l'état fondamental singulet non-magnétique et de l'ouverture d'un gap $\Delta_{s p} \approx 1.76 k T_{S P}$ dans le spectre des excitations magnétiques. De façon intéressante mais spéculative, les interactions spin-réseau pourraient aussi générer des modes hybrides, couplant les degrés de liberté de réseau et magnétiques, et ainsi donner lieu à des termes INM non evanescent. Le spectre d'excitations magnétiques de $\mathrm{CuGeO}_{3}$ a été étudié en long et en large par diffusion inélastique des neutrons non-polarisés [46,47]. En accord avec toutes les données expérimentales, le gap en énergie (en fait l'énergie correspondant au maximum de la réponse magnétique en $q=\pi$ ) s'élève à environ $\Delta_{S P} \approx 2 \mathrm{meV}$ pour des positions dans l'espace réciproque correspondants aux vecteurs de diffusion antiferromagnétiques (AF) $Q=\left(0, Q_{k}, Q_{l}\right)$, avec $Q_{k}=2 k+1$ et $Q_{l}=l+1 / 2(k$ et $l$ entiers). Comme le montrent les mesures détaillées de diffusion inélastique des neutrons non-polarisés, le mode spin-Peierls présente aussi une forte dispersion selon la direction $b$ (perpendiculaire à l'axe de la chaîne), avec une énergie de bord de zone de Brillouin $\hbar \omega\left(q_{b}=1\right) \approx 5.6 \mathrm{meV}$ [46,47]. Les mesures complètes en APL sur $\mathrm{CuGeO}_{3}$ ont permis de déterminer séparément les différentes composantes magnétiques et (possiblement) structurales mises en jeu dans le mode spin-Peierls [43,44]. La Figure 12 montre le même scan à $\mathbf{Q}$ constant réalisé au vecteur de diffusion $\mathbf{Q}=(0,-1,1 / 2)$ en $\mathrm{SF}$ et NSF pour une polarisation initiale $\mathrm{P}_{0}$ appliquée successivement selon $\mathbf{x}\left(\mathrm{P}_{x x}\right), \mathbf{y}\left(\mathrm{P}_{y y}\right)$ et $\mathbf{z}\left(\mathrm{P}_{z z}\right)$. Comme attendu pour un mode purement magnétique, le mode à $2 \mathrm{meV}$ apparaît presque complètement $\mathrm{SF}$ pour $\mathbf{P}_{0} \| \mathbf{Q}$ (on a eneffet $\left.P_{x x} \approx-P_{0}\right)$ et presque entièrement dépolarisé pour $\mathbf{P}_{0} \perp \mathbf{Q}\left(P_{y y} \approx P_{z z} \approx 0\right.$ ). Ces résultats montrent, entre autre chose, la faible anisotropie des fonctions de corrélations spin-spin, un résultat en contradiction avec la relativement forte anisotropie du tenseur $\mathrm{g}$ observé par mesures de susceptibilité. Si on néglige les termes INM et le terme chiral supposés petits, il est possible d'utiliser 

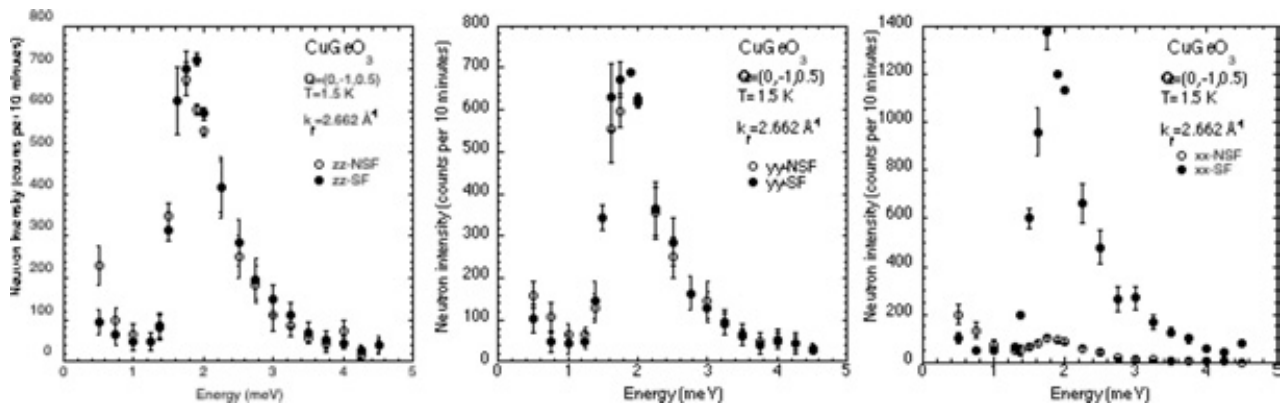

Figure 12. Scan à $\mathbf{Q}$ constant en $\mathbf{Q}=(0,-1,1 / 2)$ montrant les contributions NSF et SF dans les trois configurations avec $\mathbf{P}_{0} \|$ et $\perp \mathbf{Q}$.

l'équation (3.16) qui relie la contribution nucléaire aux trois polarisations longitudinales $P_{x x}, P_{y y}$ et $P_{z z}$ pour tenter de déterminer la contribution nucléaire sous-jacente. Pour $\mathrm{CuGeO}_{3}$, la mesure des composantes longitudinales donnent $P_{x x} \approx-0.870(5), P_{y y} \approx 0.000(5)$ et $P_{z z} \approx-0.030(5)$ [62], impliquant un rapport $\frac{N}{M_{y y}+M_{z z}} \approx 0.001 \pm 0.002$ qui confirme quantitativement la nature essentiellement magnétique du mode spin-Peierls à $2 \mathrm{meV}$, au moins pour le vecteur d'onde étudié. Ce résultat est en accord avec le modèle standard de la transition spin-Peierls dans la limite des couplages faibles [52], bien qu'il ait été prouvait que ce modèle n'était pas le meilleur pour décrire la transition de phase dans $\mathrm{CuGeO}_{3}$.

\subsection{Contributions chirales dans $\mathrm{Sr}_{14} \mathrm{Cu}_{24} \mathrm{O}_{41}$ sous champ magnétique}

Dans cette section nous allons illustrer l'interêt de l'APL pour la caractérisation et finalement la compréhension de la nature des excitations magnétiques dans les systèmes à électrons corrélés présentant un gap quantique, comme par exemple $\mathrm{Sr}_{14} \mathrm{Cu}_{24} \mathrm{O}_{41}$. Le cuprate $\mathrm{Sr}_{14} \mathrm{Cu}_{24} \mathrm{O}_{41}$ est un matériau fascinant dont la structure cristallographique peut se décrite comme un empilement imparfait de plans de deux systèmes de spin $S=1 / 2$ différents : des chaînes linéaires $\mathrm{CuO}_{2}$ et des échelles de spins à deux montants

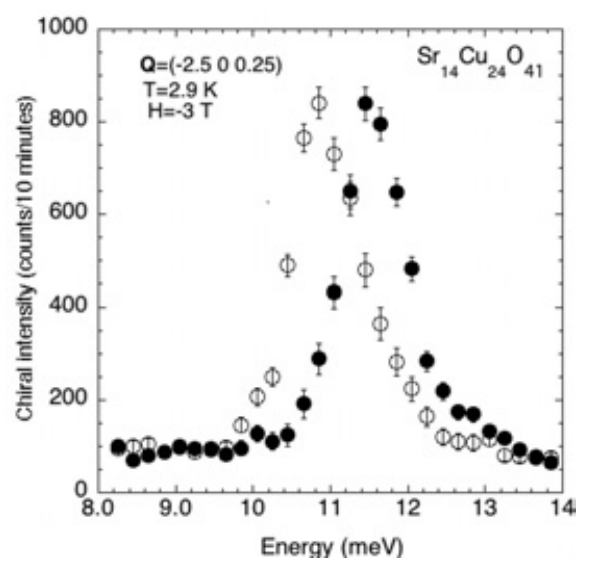

Figure 13. Analyse de polarisation de la diffusion inélastique d'un faisceau de neutrons non-polarisés sur le mode dans les chaînes $\mathrm{CuO}_{2}$ de $\mathrm{Sr}_{14} \mathrm{Cu}_{24} \mathrm{O}_{41}$ en $\mathbf{Q}=(-2.5,0,0.25)$, sous un champ magnétique de $3 \mathrm{~T}$. Les symboles vides et pleins représentent respectivement les états finaux $|-\rangle$ et $|+\rangle$ du spin des neutrons. 
$\mathrm{Cu}_{2} \mathrm{O}_{3}$ [53]. Ce composé contient naturellement une grande quantité de trous (environ 0.6 trous/Cu), qui se trouvent principalement localisés dans les chaînes $\mathrm{CuO}_{2}$ [54-56]. Par substitution des ions $\mathrm{Ca}^{2+}$ par du Sr${ }^{2+}$, les trous sont transférés des chaînes vers les échelles [55,56], le matériau subissant une transition isolant-métal à pression ambiante [54-57] et devenant même supraconducteur au dessus de $3 \mathrm{Gpa}$ [58]. Toute la question est bien évidemment de comprendre le mécanisme de l'apparition de cette supraconductivité : BCS ou non-conventionnelle? Les spectres d'excitations magnétiques dans les chaînes [60] et les échelles [23,59] ont été étudiés en détail par diffusion inélastique des neutrons. En particulier, les gaps associés aux diverses transitions singulet - triplet ont pu être déterminés avec précision : 10-12 meV pour les chaînes [60] et 32-33 meV pour les échelles [59]. En accord avec les mesures de susceptibilité et de transport [54], le gap associé aux chaînes a été attribué à l'existence de dimères de spin 1/2, $\uparrow 0 \downarrow$ (spin-trou-spin), faiblement corrélés, tandis que les trous apparaissent comme ordonnés à longue distance à basse température, formant ainsi un cristal de trous. Comme expliqué précédemment, la contribution inélastique chirale peut être déterminée à partir de la mesure de la polarisation après diffusion, en partant d'un faisceau incident non polarisé et en appliquant un champ magnétique parallèlement au vecteur de diffusion. Dans une telle configuration, l'état triplet excité à $q=\pi$ doit se splitter linéairement sous champ en deux modes d'énergies $\Delta_{0} \pm g \mu_{B} H$, tandis que le troisième mode (constant en énergie et associé à des composantes de spin parallèles au champ et à Q) ne devrait pas contribuer à la diffusion magnétique (en vertu de la règle de sélection de la diffusion magnétique). La Figure 13 résume l'analyse de polarisation de la diffusion inélastique en $\mathbf{Q}=(-2.5,0,0.25)$, sous un champ magnétique appliqué de $3 \mathrm{~T}$ [61]. Entre autre, les résultats ci-dessus confirment le décalage de $\pm g \mu_{B} H \approx \pm 0.3$ $\mathrm{meV}$ du gap associé aux chaînes de part et d'autre de $11.2 \mathrm{meV}$, la valeur du gap en champ nul. Ils démontrent en tout cas la nature totalement SF de la diffusion observée. Le faisceau de neutrons apparaît ainsi complètement polarisée, ce qui implique nécessairement d'une part l'existence d'un terme chiral assez fort et d'autre part une brisure forte, sinon totale, de la symétrie par renversement du temps [61]. La dépendance en énergie de la contribution chirale, que reflète directement la différence $I_{+}-I_{-}$, est présentée sur la Figure 14. En accord avec la discussion du paragraphe 3.2, la contribution chirale doit être une fonction impaire de l'énergie, centrée autour de la valeur du gap. En accord avec les expressions générales (3.1) et (3.2) discutées précédemment, l'existence d'un terme chiral fort doit nécessairement refléter l'existence de fonctions de corrélations spin-spin fortes couplant les deux composantes de spin (transverses) selon les directions y et $\mathbf{z}$, avec qualitativement $\left\langle S_{y} S_{z}\right\rangle \sim\left\langle S_{y} S_{y}\right\rangle \sim\left\langle S_{z} S_{z}\right\rangle$. Un tel comportement peut être facilement compris à partir de l'image très générale selon laquelle l'état excité correspondent en fait à une précession collective des spins induite par le champ et tournant dans le sens

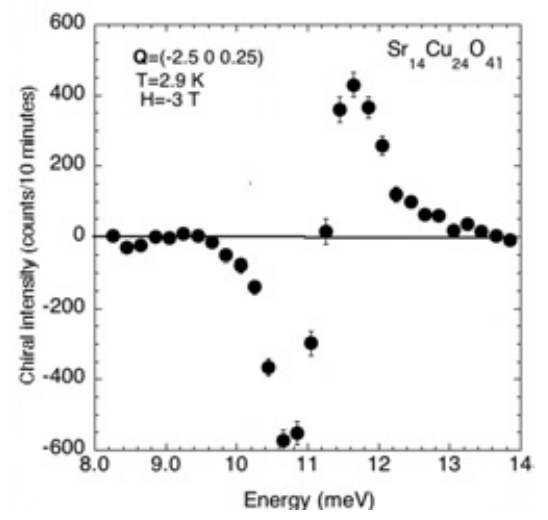

Figure 14. Dépendance en énergie de la différence des intensités associées aux états de spin $|+\rangle$ et $|-\rangle$ des neutrons en $\mathbf{Q}=(-2.5,0,0.25)$, montrant la forte contribution chirale dans $\mathrm{Sr}_{14} \mathrm{Cu}_{24} \mathrm{O}_{41}$. 
des aiguilles du montre ou inverse du sens des aiguilles d'une montre autour de la direction du champ, avec une pulsation de Larmor $\pm g \mu_{B} H / \hbar$. Cet exemple illustre très bien le mécanisme de brisure de la symétrie par renversement du temps induit par un champ magnétique.

\subsection{Polarimétrie sphérique sur $\mathrm{CuGeO}_{3}$}

Dans cette section, nous allons montrer comment la polarimétrie neutronique spérique peut aider à résoudre le problème complèt, c'est-à-dire déterminer le jeu complet de fonctions de corrélation impliquant les degrés de liberté nucléaires et magnétiques. Le composé spin-Peierls $\mathrm{CuGeO}_{3}$, dans lequel les interactions spin-réseau sont sencées être fortes, apparait à priori comme un bon candidat pour tenter une telle détermination. En section 4.5, on a montré que le facteur de structure nucléaire associé à l'hypothétique mode hybride était très petit, puisqu'il représentait au mieux $0.3 \%$ du facteur de structure magnétique principal à $q=\pi$. Si par chance les fonctions de corrélation hybrides mixant les degrés de liberté magnétiques et structuraux étaient, elles, suffisamment fortes, elles pourraient donner naissance à un terme INM d'amplitude petite, certe, mais non nulle, qui pourrait produire une rotation mesurable du vecteur polarisation. Maleyev [9] et Cepas et al. [64] ont considéré le cas théorique d'un système spin-Peierls de spin $S=1 / 2$ décrit par un Hamiltonien mettant en jeux des interactions dynamiques de Heisenberg $(\mathrm{H})$ et de Dzyaloshinski-Moriya (DM), toutes deux données par le terme générique :

$$
\sum_{i, j} \sum_{\alpha, \beta} V^{\alpha \beta}\left(\mathbf{R}_{j}-\mathbf{R}_{i}\right) S_{i}^{\alpha} S_{j}^{\beta},
$$

où $V^{\alpha \beta}\left(\mathbf{R}_{j}-\mathbf{R}_{i}\right)=J_{\beta}\left(\mathbf{R}_{j}-\mathbf{R}_{i}\right) \delta_{\alpha \beta}$ pour le premier et $\Sigma_{\gamma} D_{\gamma}\left(\mathbf{R}_{j}-\mathbf{R}_{i}\right) \epsilon_{\gamma \alpha \beta}$ pour le second. Les coefficients $D_{\gamma}$ sont les composantes du vecteur de Dzyaloshinski-Moriya, vecteur qui dépend en fait de la position de tous les ions impliqués dans le trajet du super-échange. Maleyev [9-11], en utilisant la théorie standard des perturbations, a trouvé que les termes INM sont reliés aux fonctions de corrélation à trois spins, selon l'expression :

$$
\left\langle N_{Q}^{+} \mathbf{M}_{Q}\right\rangle_{\omega}=N^{\frac{-1}{2}} \sum_{n, i, j} b_{n} \sum_{\alpha, \beta}\left\langle e^{-i \mathbf{Q} \cdot \mathbf{R}_{n}}, V_{i j}^{\alpha \beta}\right\rangle_{\omega}\left\langle S_{i}^{\alpha} S_{j}^{\beta}, \mathbf{M}_{Q}\right\rangle_{\omega}
$$

Dans l'équation (4.3), l' amplitude des termes INM semble controlée par le premier terme entre crochets. Ce terme peut être non-nul si les excitations de réseau modulent $\mathbf{R}_{n}$ et les composantes de $V$. Pour un système ne présentant pas d'ordre à longue distance, comme c'est le cas de $\mathrm{CuGeO}_{3}$, les termes scalaires d'échange de Heisenberg ne contribuent pas aux INM et seul le terme de Dzyloshinski-Moriya peut donner un effet. D'après la théorie générale $[10,11,64]$, l'expression donnant les termes INM contient des facteurs de la forme $\frac{1}{\omega^{2}-\omega_{\lambda}^{2}}$, où $\omega_{\lambda}$ est l'énergie du phonon et $\lambda$ numérote les différentes branches de phonons (acoustiques ou optique). Ainsi, les termes INM doivent ils être a priori recherchés autour des vecteurs de diffusion pour lesquels les contributions magnétiques et nucléaires ne sont pas trop éloignées en énergie. Evidemment, l'amplitude de ces termes doit aussi dépendre de façon drastique de la polarisation du phonon impliqué dans le processus d'interférence (il faut se rappeler que lors d'une expérience de diffusion inélastique de neutrons, il n'est pas possible de mesurer simultanément les composantes de spin et les déplacements atomiques dans la même direction). Afin de vérifier ces prédictions, une analyse de polarisation sphérique du mode à $2 \mathrm{meV}$ dans $\mathrm{CuGeO}_{3}$ a été réalisée avec le dispositif CRYOPAD. Comme on l'a expliqué précédemment, la procédure expérimentale consiste à déterminer, pour chaque couple $(\mathbf{Q}, \omega)$, les 18 composantes $P_{\alpha \beta}$ et $P_{\bar{\alpha} \beta}(\alpha, \beta=x, y, z)$ de la matrice de polarisation, après avoir effectué les diverses corrections de bruit de fond appropriées. Le Table 2 donne les valeurs de $P_{\alpha \beta}$ et $P_{\bar{\alpha} \beta}(\alpha, \beta=x, y, z)$ et les barres d'erreurs correspondantes pour le mode spin-Peierls en $\mathbf{Q}=(0,1,1 / 2)[62,63]$. A partir de ce table, il est possible d'en déduire les composantes longitudinales :

$$
\bar{P}_{x x}=(-0.963 \pm 0.005) P_{0}
$$




$$
\begin{aligned}
& \bar{P}_{y y}=(0.005 \pm 0.006) P_{0} \\
& \bar{P}_{z z}=(-0.031 \pm 0.006) P_{0}
\end{aligned}
$$

Table 2. Analyse de polarisation sphérique du mode à 2 meV en $\mathbf{Q}=(0,1,1 / 2)$ dans $\mathrm{CuGeO}_{3}$. Composantes longitudinales et transverses de la matrice de polarisation.

\begin{tabular}{cccc}
\hline$\alpha \beta$ & $P_{\alpha \beta}( \pm \Delta P)$ & $\bar{\alpha} \beta$ & $P_{\bar{\alpha} \beta}( \pm \Delta P)$ \\
\hline$x x$ & $-0.868(6)$ & $\bar{x} x$ & $0.866(6)$ \\
$x y$ & $-0.034(8)$ & $\bar{x} y$ & $0.044(8)$ \\
$x z$ & $-0.015(8)$ & $\bar{x} z$ & $0.025(8)$ \\
$y x$ & $0.004(8)$ & $\bar{y} x$ & $0.021(8)$ \\
$y y$ & $0.001(8)$ & $\bar{y} y$ & $-0.008(8)$ \\
$y z$ & $0.006(8)$ & $\bar{y} z$ & $0.001(8)$ \\
$z x$ & $0.006(8)$ & $\bar{z} x$ & $0.015(8)$ \\
$z y$ & $-0.004(8)$ & $\bar{z} y$ & $-0.004(8)$ \\
$z z$ & $-0.031(8)$ & $\bar{z} z$ & $0.026(8)$ \\
\hline
\end{tabular}

avec $P_{0}=0.89 \pm 0.005$, et où $\bar{P}_{\alpha \alpha}=\frac{P_{\alpha \alpha}-P_{\bar{\alpha} \alpha}}{2}$ est la partie antisymétrique qui élimine au premier ordre les termes indépendants de $P_{0}$. CRYOPAD permet une détermination très précise des composantes longitudinales, la limite étant principalement due à l'erreur statistique sur le comptage (l'erreur intrinsèque est plus petite que 0.0005 !). En accord avec les mesures en APL, la quantité $\frac{\bar{P}_{x x}+\bar{P}_{y y}+\bar{P}_{z z}}{P_{0}}=$ $-0.989 \pm 0.010$ est très proche de 1 , ce qui implique à nouveau pour la contribution nucléaire associée a l'hypothétique mode hybride à $q=\pi$ une très petite valeur, $\frac{N}{\sigma_{0}}=0.003 \pm 0.003$.

En appliquant le jeu de relations donné en section 3.2, il est possible d'obtenir une estimation relativement précises des amplitudes des différents termes magnétiques et INM (parties symétriques et anti-symétriques) :

$$
\begin{aligned}
& M_{y y}-M_{z z}=0.015 \pm 0.010 \\
& M_{c h} / \sigma_{0}=0 \pm 0.003 \\
& M_{y z} / \sigma_{0}=-0.003 \pm 0.003 \\
& R_{y} / \sigma_{0}=0 \pm 0.004 \\
& I_{y} / \sigma_{0}=-0.008 \pm 0.005 \\
& R_{z} / \sigma_{0}=0.004 \pm 0.004 \\
& I_{z} / \sigma_{0}=-0.015 \pm 0.005
\end{aligned}
$$

Ainsi, à la précision des mesures, le terme chiral et les termes INM symétriques apparaissent être très petits, tandis que les termes INM antisymétriques (en particuliers $I_{z}$ ) apparaissent être non nuls, bien qu'à la limite de précision de la version du CRYOPAD utlisé (environ \pm 0.01 dans le cas présent). Pour résumer les résultats de la PSN sur le mode spin-Peierls dans $\mathrm{CuGeO}_{3}$, l'amplitude des termes INM, si ils existent, est estimée à au mieux $1 \%$ du signal magnétique principal. En $q \approx \pi$ et $\hbar \omega \approx \Delta_{S P}$, les excitations magnétiques et de réseau peuvent être considérées comme complètement découplées. La raison principale de ce résultat négatif vient probablement du fait que dans $\mathrm{CuGeO}_{3}, \Delta_{S P} \ll \omega_{p h}$. Les modes hybrides pourraient éventuellement exister à d'autres valeurs de q ou de l'énergie pour lesquelles les spectres d'excitations magnétiques et structurales sont superposés, comme par exemple à celles correspondant à la limite supèrieure du continuum d'excitations magnétiques [65]. Malheureusement, malgré plusieurs tentatives, ces modes n'ont pas encore pu être détectés dans $\mathrm{CuGeO}_{3}$, probablement parce que l'Hamiltonien décrivant ce système est dominé par des interactions scalaires et non vectorielles. 


\section{CONCLUSION}

Dans ce chapitre, nous avons montré toute la puissance de la polarimétrie neutronique pour l'étude des processus magnétiques inélastiques en physique de la matière condensée. La méthode la plus fréquemment utilisée, dite Analyse de Polarisation Longitudinale (APL), est particuliérement adaptée aux études nécessitant une détermination précise des fonctions de corrélations spin-spin dynamiques impliquant seulement les composantes longitudinales du tenseur de susceptibilité généralisée. Entre autres choses, l'APL permet d'obtenir séparément les termes structuraux et les divers facteurs de structure magnétiques longitudinaux (essentiellemnt les parties dépendantes de $P_{0}$, le terme chiral, l'anisotropie des différentes fonctions de corrélations). En principe, l'APL peut aussi être utilisée pour résoudre des problèmes dans lesquels certains termes d'interférence nucléaire-magnétique peuvent exister. S'ils sont suffisamment forts, certains termes INM peuvent produire une rotation du vecteur polarisation autour du vecteur interaction différente de $\pi$, qui ne peut être déterminée que par polarimétrie neutronique sphérique (PNS). En théorie, la mesure des neuf coefficients de la matrice de polarisation devrait permettre de résoudre de façon non ambigüe le problème et finalement, devrait permettre de déterminer les différentes fonctions de corrélations nucléaire-nucléaire, magnétique-magnétique et nucléaire-magnétique.

\section{Références}

[1] Halpern, O., and Johnson, M.H., Phys. Rev. 55, 898 (1939).

[2] Halpern, O., and Holstein, T., Phys. Rev. 59, 960 (1941).

[3] Maleyev, S.V., Sov. Phys. JETP 13, 595 (1961).

[4] Izyumov, Yu., and Maleyev, S.V., Sov. Phys. JETP 14, 1168 (1962).

[5] Izyumov, Yu., Sov. Phys. JETP 15, 1167 (1962).

[6] Maleyev, S.V., Baryakhtar, V.G., and Suris, A., Sov. Phys. Solid State 4, 2533 (1963).

[7] Blume, M., Phys. Rev. 130, 1670 (1963). and references therein.

[8] Schermer, R.I., and Blume, M., Phys. Rev. 166, 554 (1968).

[9] Maleyev, S.V., Physica B 267-268, 236 (1999).

[10] Maleyev, S.V., Physica B 297, 67 (2001)

[11] Maleyev, S.V., Physics-Uspekhi 45, 569 (2002). and references therein

[12] Trammell, G.T., Phys. Rev. 92, 1387 (1953).

[13] Lovesey, S.W., in Theory of Neutron Scattering from Condensed Matter, vol. 1 and 2, Clarendon Press, Oxford, 1987.

[14] Balcar, E., and Lovesey, S.W., in Theory of Magnetic Neutron and Photon Scattering, Clarendon Press, Oxford, 1989.

[15] Van Hove, L., Phys. Rev. 95, 249 (1954).

[16] Schwinger, J., Phys. Rev. 73, 407 (1948).

[17] Blume, M., Phys. Rev. 133, A1366 (1964).

[18] Squires, G. L., in Introduction to the theory of thermal neutron scattering, Dover publications, Mineola, New York, 1986.

[19] Moon, R.M., Riste, T., and Koehler, W.C., Phys. Rev. 181,920 (1969).

[20] Drabkin, G.M., et al., JETP Lett. 2, 353 (1965).

[21] Brehmer, S., et al., Phys. Rev. B 60, 329 (1999).

[22] Muller, M., et al., Phys. Rev. B 66, 34423 (2002).

[23] Matsuda, M., et al., Phys. Rev. B 62, 8903 (2000).

[24] Maleyev, S.V., Phys. Rev. Lett. 75, 4682 (1995) and references therein.

[25] Aristov, D.N., and Maleyev, S.V., Phys. Rev. B 62, R751 (2000) and references therein.

[26] Tasset, F., Physica B 156-157, 627 (1989). 
[27] Brown, P.J., Forsyth, J.B., and Tasset, F., Proc. R. Soc. London A 442, 147 (1993).

[28] Rossat-Mignod, J., Burlet, P., Quezel, S., and Vogt, O., Physica B 102, 237 (1980).

[29] Bartholin, H., Florence, D., Wang Tcheng-Si, and Vogt, O., Phys. Status Solidi A 24, 631 (1974).

[30] Rossat-Mignod, J., Burlet, P., Villain, J., Bartholin, H., Wang Tcheng-Si, Florence, D., and Vogt, O., Phys. Rev. B 16, 440 (1977).

[31] Takahashi, H., and Kasuya, T., J. Phys. C 18, 2697 (1985).

[32] Regnault, L.P., Jacoud, J.L., Vettier, C., Chattopadhyay, T., Rossat-Mignod, J., Suzuki, T., Kasuya, T., and Vogt, O., Physica B 156 \& 157, 798 (1989).

[33] Mikeska, H., J. Phys. C 13, 2913 (1980).

[34] Maki, K., J. Low. Temp. Phys. 41, 327 (1981).

[35] Regnault, L.P., Boucher, J.P., Rossat-Mignod, J., Renard, J.P., Bouillot, J., and Stirling, W.G., J. Phys. C 15,1261 (1982) and references therein.

[36] Boucher, J.P., Regnault, L.P., Pynn, R., Bouillot, J., and Renard, J.P., Europhys. Lett. 1, 415 (1986) and references therein.

[37] Sasaki, K., in Proceedings of the VII Kyoto Summer Institute, edited by S. Takeno (Springer, Berlin, 1985).

[38] Vaknin, D., et al., Phys. Rev. Lett. 58, 2802 (1987).

[39] Rossat-Mignod, J., et al., Physica C 185-189, 86 (1991).

[40] Fong, H.F., et al., Phys. Rev. Lett. 75, 316 (1995); H. F. Fong et al., Nature 398, 588 (1999).

[41] Regnault, L.P., Bourges, P., and Burlet, P., in Neutron Scattering in Layered Copper-oxyde Superconductors, edited by A. Furrer (Kluwer Academic Publishers, DORDRECHT/BOSTON/ LONDON, 1998) p. 85-134 and references therein.

[42] Hayden, S., in Neutron Scattering in Layered Copper-oxyde Superconductors, edited by A. Furrer (Kluwer Academic Publishers, DORDRECHT/BOSTON/LONDON, 1998) p. 135-164 and references therein.

[43] Regnault, L.P., et al., Physica B 335, 19-25 (2003).

[44] Regnault, L.P., et al., Physica B 345, 111-118 (2004).

[45] Hase, M., Terasaki, I., and Uchinokura, K., Phys. Rev. Lett. 70, 3651 (1993).

[46] Nishi, M., Fujita, O., and Akimitsu, J., Phys. Rev. B 50, 6508 (1994).

[47] Regnault, L.P., AŢn, M., Hennion, B., Dhalenne, G., and Revcolevschi, A., Phys. Rev. B 53, 5579 (1996) and references therein.

[48] Pouget, J.P., Regnault, L.P., Aïn, M., Hennion, B., Renard, J.P., Veillet, P., Dhalenne, G., and Revcolevschi, A., Phys. Rev. Lett. 72, 4037 (1994). Phys. Rev. Lett. 78, 1560 (1997).

[49] Boucher, J.P., and Regnault, L.P., J. Phys. I, France, 6, 1939 (1996) and references therein.

[50] Lorenzo, J.E., Regnault, L.P., Hennion, B., Aïn, A., Bourdarot, F., Kulda, J., Dhalenne, G., and Revcolevschi, A., J. Phys. : Condensed Matter 9, L211 (1997).

[51] Bouzerar, G., Legeza, O., and Ziman, T., Phys. Rev. B 60, 15278 (1999).

[52] Pytte, E., Phys. Rev. B 10, 4637 (1974); Cross, M.C., and Fischer, D.S., Phys. Rev. B 19, 402 (1979); Bray, J.W., Interrante, L.V., Jacobs, I.S., and Bonner, J.C., in Extended Linear Chain Compounds, edited by Miller, J.S., (Plenum, New York, 1982), Vol. 3, pp. 353-415.

[53] McCaron, E.M., Subramaniam, M.A., Calabrese, J.C., and Harlow, R.L., Mater. Res. Bull. 23, 1355 (1988).

[54] Kato, M., Shiota, K., and Koike, Y., Physica C 258, 284 (1996).

[55] Mizuno, Y., Tohyama, T., and Maekawa, S., J. Phys. Soc. Jpn 66, 937 (1997).

[56] Osafune, T., Motoyama, N., Eisaki, H., and Uchida, S., Phys. Rev. Lett. 78, 1980 (1997).

[57] Motoyama, N., Osafune, T., Kakeshita, T., Eisaki, H., and Uchida, S., Phys. Rev. B 55, 3386 (1997). 
[58] Uehara, M., Nagata, T., Akimitsu, J., Takahashi, H., Môri, N., and Kinoshita, K., J. Phys. Soc. Jpn. 65, 2764 (1997).

[59] Matsuda, M., and Katsumata. K., Phys. Rev. B 53, 12201 (1996).

[60] Regnault, L.P., Boucher, J.P., Moudden, H., Lorenzo, J.E., Hiess, A., Ammerahl, U., Dhalenne, G., and Revcoloevschi, A., Phys. Rev. B 59, 1055 (1999).

[61] Boullier, C., Regnault, L.P., Lorenzo, J.E., Dhalenne, G., and Revcoloevschi, A., to be published.

[62] Regnault, L.P., Ronnow, H.M., Lorenzo, J.E., Bellissent, R., and Tasset, F., Physica B 335, 19-25 (2003).

[63] Regnault, L.P., Ronnow, H.M., Boullier, C., Lorenzo, J.E., and Marin, C., Physica B 345, 111-118 (2004).

[64] Cepas, O., and Ziman, T., in: Quantum properties of low-dimensional antiferromagnets, eds. Ajiro, Y. and Boucher, J.P., (Kyushu University Press, 2002), pp.175-182.

[65] Braden, M., Hennion, B., Pfeuty, P., Dhalenne, G., and Revcolevschi, A., Phys. Rev. Lett. 83, 1858 (1999). 